Chemical and Structural Diversity in Chiral Magnesium Tartrates and Their Racemic and Meso Analogues

\author{
Kinson C. Kam, Karen L. M. Young, and Anthony K. Cheetham
}

$\underline{\text { Supporting Information }}$ 
Table S1. Atomic coordinates $\left(\times 10^{4}\right)$ and equivalent isotropic displacement parameters $\left(\AA^{2} \times 10^{3}\right)$ for $\left[\mathrm{Mg}\left(\mathrm{D}, \mathrm{L}-\mathrm{C}_{4} \mathrm{H}_{4} \mathrm{O}_{6}\right)\left(\mathrm{H}_{2} \mathbf{O}\right)_{2}\right] \cdot \mathbf{3} \mathrm{H}_{2} \mathbf{O}, \mathbf{1}$.

\begin{tabular}{lllll}
\hline Atom & $\mathrm{X}$ & $\mathrm{y}$ & $\mathrm{z}$ & $\mathrm{U}(\mathrm{eq})$ \\
\hline $\mathrm{C}(1)$ & $3414(2)$ & $1275(2)$ & $6765(1)$ & $28(1)$ \\
$\mathrm{C}(2)$ & $2426(2)$ & $1878(2)$ & $6794(1)$ & $27(1)$ \\
$\mathrm{C}(3)$ & $4300(2)$ & $1235(2)$ & $10872(1)$ & $32(1)$ \\
$\mathrm{C}(4)$ & $4303(2)$ & $1882(2)$ & $9896(1)$ & $31(1)$ \\
$\mathrm{Mg}(1)$ & 2500 & $394(1)$ & 5000 & $29(1)$ \\
$\mathrm{Mg}(2)$ & 2500 & $435(1)$ & 10000 & $36(1)$ \\
$\mathrm{O}(1)$ & $3584(1)$ & $643(1)$ & $6048(1)$ & $34(1)$ \\
$\mathrm{O}(2)$ & $3993(1)$ & $1452(1)$ & $7466(1)$ & $37(1)$ \\
$\mathrm{O}(3)$ & $1848(1)$ & $1549(1)$ & $5958(1)$ & $30(1)$ \\
$\mathrm{O}(4)$ & $3190(2)$ & $-815(2)$ & $4245(1)$ & $54(1)$ \\
$\mathrm{O}(5)$ & $3569(1)$ & $634(1)$ & $11042(1)$ & $41(1)$ \\
$\mathrm{O}(6)$ & $5018(1)$ & $1352(1)$ & $11427(1)$ & $44(1)$ \\
$\mathrm{O}(7)$ & $3456(1)$ & $1586(1)$ & $9339(1)$ & $36(1)$ \\
$\mathrm{O}(8)$ & $3184(2)$ & $-772(2)$ & $9230(2)$ & $79(1)$ \\
$\mathrm{O}(9)$ & 7500 & 2500 & $1792(2)$ & $68(1)$ \\
$\mathrm{O}(10)$ & $6024(2)$ & $1057(2)$ & $7585(1)$ & $45(1)$ \\
$\mathrm{O}(11)$ & $4244(2)$ & 7500 & 5000 & $55(1)$ \\
$\mathrm{O}(12)$ & $4982(1)$ & $8851(2)$ & $6517(1)$ & $44(1)$ \\
\hline
\end{tabular}


Table S2. Bond lengths $[\AA]$ and bond angles $\left[^{\circ}\right]$ for $\left[\mathrm{Mg}\left(\mathrm{D}, \mathrm{L}-\mathrm{C}_{4} \mathrm{H}_{4} \mathrm{O}_{6}\right)\left(\mathrm{H}_{2} \mathbf{O}\right)\right] \cdot \mathbf{3} \mathrm{H}_{2} \mathrm{O}, \mathbf{1}$.

\begin{tabular}{|c|c|c|c|}
\hline Moiety & Distance $(\AA)$ & Moiety & Distance $(\AA)$ \\
\hline $\operatorname{Mg}(1)-\mathrm{O}(4)^{\# 3}$ & $2.0149(19)$ & $\operatorname{Mg}(2)-O(8)^{\# 4}$ & $2.020(2)$ \\
\hline $\operatorname{Mg}(1)-\mathrm{O}(4)$ & $2.0149(19)$ & $\operatorname{Mg}(2)-\mathrm{O}(8)$ & $2.020(2)$ \\
\hline $\mathrm{Mg}(1)-\mathrm{O}(1)$ & $2.0624(15)$ & $\operatorname{Mg}(2)-\mathrm{O}(5)$ & $2.0329(16)$ \\
\hline $\operatorname{Mg}(1)-O(1)^{\# 3}$ & $2.0624(15)$ & $\operatorname{Mg}(2)-O(5){ }^{\# 4}$ & $2.0329(16)$ \\
\hline $\operatorname{Mg}(1)-\mathrm{O}(3)$ & $2.1006(16)$ & $\operatorname{Mg}(2)-\mathrm{O}(7)$ & $2.1030(18)$ \\
\hline $\operatorname{Mg}(1)-\mathrm{O}(3)^{\# 3}$ & $2.1006(16)$ & $\operatorname{Mg}(2)-O(7)^{\# 4}$ & $2.1030(18)$ \\
\hline \multicolumn{4}{|c|}{ Organic and Water } \\
\hline Moiety & Distance $(\AA)$ & Moiety & Distance $(\AA)$ \\
\hline $\mathrm{C}(1)-\mathrm{O}(2)$ & $1.248(2)$ & $\mathrm{O}(3)-\mathrm{H}(3)$ & $0.89(3)$ \\
\hline $\mathrm{C}(1)-\mathrm{O}(1)$ & $1.255(2)$ & $\mathrm{O}(4)-\mathrm{H}(41)$ & $0.72(3)$ \\
\hline $\mathrm{C}(1)-\mathrm{C}(2)$ & $1.528(3)$ & $\mathrm{O}(4)-\mathrm{H}(42)$ & $0.87(3)$ \\
\hline $\mathrm{C}(2)-\mathrm{O}(3)$ & $1.430(2)$ & $\mathrm{O}(7)-\mathrm{H}(7)$ & $0.75(3)$ \\
\hline$C(2)-C(2)^{\# 1}$ & $1.522(4)$ & $\mathrm{O}(8)-\mathrm{H}(81)$ & $0.82(5)$ \\
\hline $\mathrm{C}(2)-\mathrm{H}(2)$ & $0.960(19)$ & $\mathrm{O}(8)-\mathrm{H}(82)$ & $0.74(4)$ \\
\hline $\mathrm{C}(3)-\mathrm{O}(6)$ & $1.237(3)$ & $\mathrm{O}(9)-\mathrm{H}(91)$ & $0.91(3)$ \\
\hline $\mathrm{C}(3)-\mathrm{O}(5)$ & $1.253(3)$ & $\mathrm{O}(10)-\mathrm{H}(101)$ & $0.85(4)$ \\
\hline$C(3)-C(4)$ & $1.531(3)$ & $\mathrm{O}(10)-\mathrm{H}(102)$ & $0.81(4)$ \\
\hline $\mathrm{C}(4)-\mathrm{O}(7)$ & $1.420(2)$ & $\mathrm{O}(11)-\mathrm{H}(111)$ & $0.78(3)$ \\
\hline $\mathrm{C}(4)-\mathrm{C}(4)^{\# 2}$ & $1.525(4)$ & $\mathrm{O}(12)-\mathrm{H}(121)$ & $0.78(4)$ \\
\hline $\mathrm{C}(4)-\mathrm{H}(4)$ & $0.96(2)$ & $\mathrm{O}(12)-\mathrm{H}(122)$ & $0.87(3)$ \\
\hline Moiety & Angle $\left(^{\circ}\right)$ & Moiety & Angle $\left(^{\circ}\right)$ \\
\hline $\mathrm{O}(4)^{\# 3}-\mathrm{Mg}(1)-\mathrm{O}(4)$ & $86.67(13)$ & $\mathrm{O}(5)-\mathrm{Mg}(2)-\mathrm{O}(5)^{\# 4}$ & $166.39(10)$ \\
\hline $\mathrm{O}(4)^{\# 3}-\mathrm{Mg}(1)-\mathrm{O}(1)$ & $95.28(8)$ & $\mathrm{O}(8)^{\# 4}-\mathrm{Mg}(2)-\mathrm{O}(7)$ & $169.02(10)$ \\
\hline $\mathrm{O}(4)-\mathrm{Mg}(1)-\mathrm{O}(1)$ & $96.93(8)$ & $\mathrm{O}(8)-\mathrm{Mg}(2)-\mathrm{O}(7)$ & $88.84(10)$ \\
\hline $\mathrm{O}(4)^{\# 3}-\mathrm{Mg}(1)-\mathrm{O}(1)^{\# 3}$ & $96.93(8)$ & $\mathrm{O}(5)-\mathrm{Mg}(2)-\mathrm{O}(7)$ & $76.92(6)$ \\
\hline $\mathrm{O}(4)-\mathrm{Mg}(1)-\mathrm{O}(1)^{\# 3}$ & $95.28(8)$ & $\mathrm{O}(5)^{\# 4}-\mathrm{Mg}(2)-\mathrm{O}(7)$ & $93.96(7)$ \\
\hline $\mathrm{O}(1)-\mathrm{Mg}(1)-\mathrm{O}(1)^{\# 3}$ & $163.19(9)$ & $\mathrm{O}(8)^{\# 4}-\mathrm{Mg}(2)-\mathrm{O}(7)^{\# 4}$ & $88.84(10)$ \\
\hline $\mathrm{O}(4)^{\# 3}-\mathrm{Mg}(1)-\mathrm{O}(3)$ & $88.78(8)$ & $\mathrm{O}(8)-\mathrm{Mg}(2)-\mathrm{O}(7)^{\# 4}$ & $169.02(10)$ \\
\hline $\mathrm{O}(4)-\mathrm{Mg}(1)-\mathrm{O}(3)$ & $172.41(7)$ & $\mathrm{O}(5)-\mathrm{Mg}(2)-\mathrm{O}(7)^{\# 4}$ & $93.96(7)$ \\
\hline $\mathrm{O}(1)-\mathrm{Mg}(1)-\mathrm{O}(3)$ & $77.43(6)$ & $\mathrm{O}(5)^{\# 4}-\mathrm{Mg}(2)-\mathrm{O}(7)^{\# 4}$ & $76.92(6)$ \\
\hline $\mathrm{O}(1)^{\# 3}-\mathrm{Mg}(1)-\mathrm{O}(3)$ & $91.30(6)$ & $\mathrm{O}(7)-\mathrm{Mg}(2)-\mathrm{O}(7)^{\# 4}$ & $96.87(10)$ \\
\hline $\mathrm{O}(4)^{\# 3}-\mathrm{Mg}(1)-\mathrm{O}(3)^{\# 3}$ & $172.41(7)$ & $\mathrm{C}(1)-\mathrm{O}(1)-\mathrm{Mg}(1)$ & $119.01(13)$ \\
\hline $\mathrm{O}(4)-\mathrm{Mg}(1)-\mathrm{O}(3)^{\# 3}$ & $88.78(8)$ & $\mathrm{C}(2)-\mathrm{O}(3)-\mathrm{Mg}(1)$ & $116.02(12)$ \\
\hline $\mathrm{O}(1)-\mathrm{Mg}(1)-\mathrm{O}(3)^{\# 3}$ & $91.30(6)$ & $\mathrm{Mg}(1)-\mathrm{O}(3)-\mathrm{H}(3)$ & $113.4(16)$ \\
\hline $\mathrm{O}(1)^{\# 3}-\mathrm{Mg}(1)-\mathrm{O}(3)^{\# 3}$ & $77.43(6)$ & $\mathrm{Mg}(1)-\mathrm{O}(4)-\mathrm{H}(41)$ & $127(3)$ \\
\hline $\mathrm{O}(3)-\mathrm{Mg}(1)-\mathrm{O}(3)^{\# 3}$ & $96.36(9)$ & $\mathrm{Mg}(1)-\mathrm{O}(4)-\mathrm{H}(42)$ & $124(2)$ \\
\hline $\mathrm{O}(8)^{\# 4}-\mathrm{Mg}(2)-\mathrm{O}(8)$ & $87.13(18)$ & $\mathrm{Mg}(2)-\mathrm{O}(7)-\mathrm{H}(7)$ & $124(2)$ \\
\hline $\mathrm{O}(8)^{\# 4}-\mathrm{Mg}(2)-\mathrm{O}(5)$ & $93.39(10)$ & $\mathrm{Mg}(2)-\mathrm{O}(8)-\mathrm{H}(81)$ & $126(3)$ \\
\hline $\mathrm{O}(8)-\mathrm{Mg}(2)-\mathrm{O}(5)$ & $96.47(10)$ & $\mathrm{Mg}(2)-\mathrm{O}(8)-\mathrm{H}(82)$ & $119(3)$ \\
\hline $\mathrm{O}(8)^{\# 4}-\mathrm{Mg}(2)-\mathrm{O}(5)^{\# 4}$ & $96.47(10)$ & $\mathrm{C}(3)-\mathrm{O}(5)-\mathrm{Mg}(2)$ & $120.54(13)$ \\
\hline $\mathrm{O}(8)-\mathrm{Mg}(2)-\mathrm{O}(5)^{\# 4}$ & $93.39(10)$ & $\mathrm{C}(4)-\mathrm{O}(7)-\mathrm{Mg}(2)$ & $116.33(12)$ \\
\hline
\end{tabular}




\begin{tabular}{|c|c|c|c|}
\hline \multicolumn{4}{|c|}{ Organic } \\
\hline Moiety & Angle $\left(^{\circ}\right)$ & Moiety & Angle $\left(^{\circ}\right)$ \\
\hline $\mathrm{O}(2)-\mathrm{C}(1)-\mathrm{O}(1)$ & $124.87(19)$ & $\mathrm{O}(6)-\mathrm{C}(3)-\mathrm{C}(4)$ & $117.28(19)$ \\
\hline $\mathrm{O}(2)-\mathrm{C}(1)-\mathrm{C}(2)$ & $116.85(17)$ & $\mathrm{O}(5)-\mathrm{C}(3)-\mathrm{C}(4)$ & $117.23(18)$ \\
\hline $\mathrm{O}(1)-\mathrm{C}(1)-\mathrm{C}(2)$ & $118.29(17)$ & $\mathrm{O}(7)-\mathrm{C}(4)-\mathrm{C}(4)^{\# 2}$ & $110.20(13)$ \\
\hline $\mathrm{O}(3)-\mathrm{C}(2)-\mathrm{C}(2)^{\# 1}$ & $110.38(14)$ & $\mathrm{O}(7)-\mathrm{C}(4)-\mathrm{C}(3)$ & $108.79(17)$ \\
\hline $\mathrm{O}(3)-\mathrm{C}(2)-\mathrm{C}(1)$ & $109.17(15)$ & $C(4)^{\# 2}-C(4)-C(3)$ & $110.3(2)$ \\
\hline$C(2)^{\# 1}-C(2)-C(1)$ & $111.0(2)$ & $\mathrm{O}(7)-\mathrm{C}(4)-\mathrm{H}(4)$ & $112.0(11)$ \\
\hline $\mathrm{O}(3)-\mathrm{C}(2)-\mathrm{H}(2)$ & $110.3(12)$ & $\mathrm{C}(4)^{\# 2}-\mathrm{C}(4)-\mathrm{H}(4)$ & $108.2(12)$ \\
\hline $\mathrm{C}(2)^{\# 1}-\mathrm{C}(2)-\mathrm{H}(2)$ & $105.9(12)$ & $\mathrm{C}(3)-\mathrm{C}(4)-\mathrm{H}(4)$ & $107.3(11)$ \\
\hline $\mathrm{C}(1)-\mathrm{C}(2)-\mathrm{H}(2)$ & $110.0(12)$ & $\mathrm{C}(2)-\mathrm{O}(3)-\mathrm{H}(3)$ & $111.5(16)$ \\
\hline $\mathrm{O}(6)-\mathrm{C}(3)-\mathrm{O}(5)$ & $125.49(19)$ & $\mathrm{C}(4)-\mathrm{O}(7)-\mathrm{H}(7)$ & $109(2)$ \\
\hline \multicolumn{4}{|c|}{ Water } \\
\hline Moiety & Angle $\left(^{\circ}\right)$ & Moiety & Angle $\left(^{\circ}\right)$ \\
\hline $\mathrm{H}(41)-\mathrm{O}(4)-\mathrm{H}(42)$ & $105(3)$ & $\mathrm{H}(101)-\mathrm{O}(10)-\mathrm{H}(102)$ & $102(3)$ \\
\hline $\mathrm{H}(81)-\mathrm{O}(8)-\mathrm{H}(82)$ & $114(4)$ & $\mathrm{H}(121)-\mathrm{O}(12)-\mathrm{H}(122)$ & 104(3) \\
\hline
\end{tabular}

Symmetry transformations used to generate equivalent atoms:

$\# 1-\mathrm{x}+1 / 2,-\mathrm{y}+1 / 2, \mathrm{z} \quad \# 2 \mathrm{x},-\mathrm{y}+1 / 2,-\mathrm{z}+2 \quad \# 3-\mathrm{x}+1 / 2, \mathrm{y},-\mathrm{z}+1 \quad \# 4-\mathrm{x}+1 / 2, \mathrm{y},-\mathrm{z}+2$ 
Table S3. Atomic coordinates $\left(\mathrm{x}_{10} 0^{4}\right.$ ) and equivalent isotropic displacement parameters $\left(\AA^{2} \times 10^{3}\right)$ for $\left[\mathbf{M g}\left(\mathbf{D}, \mathbf{L}-\mathbf{C}_{4} \mathbf{H}_{4} \mathbf{O}_{6}\right)\left(\mathbf{H}_{2} \mathbf{O}\right)\right] \cdot \mathbf{H}_{2} \mathbf{O}, \mathbf{2}$.

\begin{tabular}{lllll}
\hline Atom & $\mathrm{x}$ & $\mathrm{y}$ & $\mathrm{z}$ & $\mathrm{U}(\mathrm{eq})$ \\
\hline $\mathrm{C}(1)$ & $8581(2)$ & $10005(3)$ & $639(2)$ & $19(1)$ \\
$\mathrm{C}(2)$ & $9236(2)$ & $9756(3)$ & $2261(2)$ & $18(1)$ \\
$\mathrm{C}(3)$ & $6468(2)$ & $5601(3)$ & $3143(2)$ & $21(1)$ \\
$\mathrm{C}(4)$ & $5738(2)$ & $7398(3)$ & $2590(2)$ & $19(1)$ \\
$\mathrm{Mg}$ & $7481(1)$ & $6499(1)$ & $924(1)$ & $20(1)$ \\
$\mathrm{O}(1)$ & $7878(2)$ & $8744(2)$ & $-97(2)$ & $25(1)$ \\
$\mathrm{O}(2)$ & $8809(2)$ & $11497(2)$ & $154(2)$ & $25(1)$ \\
$\mathrm{O}(3)$ & $8782(2)$ & $8090(2)$ & $2623(2)$ & $22(1)$ \\
$\mathrm{O}(4)$ & $7197(2)$ & $4981(2)$ & $2526(2)$ & $24(1)$ \\
$\mathrm{O}(5)$ & $6285(2)$ & $4900(2)$ & $4195(2)$ & $28(1)$ \\
$\mathrm{O}(6)$ & $5894(2)$ & $7868(2)$ & $1275(2)$ & $21(1)$ \\
$\mathrm{O}(7)$ & $9131(2)$ & $4967(3)$ & $1007(2)$ & $29(1)$ \\
$\mathrm{O}(8)$ & $6191(3)$ & $1416(3)$ & $1494(3)$ & $55(1)$ \\
\hline
\end{tabular}


Table S4. Bond lengths $[\AA ̊]$ and bond angles $\left[{ }^{\circ}\right]$ for $\left[\mathbf{M g}\left(\mathbf{D}, \mathbf{L}-\mathbf{C}_{4} \mathbf{H}_{4} \mathbf{O}_{6}\right)\left(\mathbf{H}_{2} \mathbf{O}\right)\right] \cdot \mathbf{H}_{2} \mathbf{O}, \mathbf{2}$.

\begin{tabular}{|c|c|c|c|}
\hline Moiety & Distance $(\AA)$ & Moiety & Distance $(\AA)$ \\
\hline $\mathrm{Mg}-\mathrm{O}(5)^{\# 3}$ & $2.0218(17)$ & $\mathrm{Mg}-\mathrm{O}(7)$ & $2.084(2)$ \\
\hline $\mathrm{Mg}-\mathrm{O}(1)$ & $2.0652(18)$ & $\mathrm{Mg}-\mathrm{O}(3)$ & $2.1177(17)$ \\
\hline $\mathrm{Mg}-\mathrm{O}(4)$ & $2.0687(18)$ & $\mathrm{Mg}-\mathrm{O}(6)$ & $2.1261(18)$ \\
\hline \multicolumn{4}{|c|}{ Organic and Water } \\
\hline Moiety & Distance $(\AA)$ & Moiety & Distance $(\AA)$ \\
\hline $\mathrm{C}(1)-\mathrm{O}(1)$ & $1.251(3)$ & $\mathrm{C}(4)-\mathrm{O}(6)$ & $1.432(3)$ \\
\hline $\mathrm{C}(1)-\mathrm{O}(2)$ & $1.258(3)$ & $\mathrm{C}(4)-\mathrm{C}(4)^{\# 2}$ & $1.540(4)$ \\
\hline $\mathrm{C}(1)-\mathrm{C}(2)$ & $1.528(3)$ & $\mathrm{C}(4)-\mathrm{H}(4)$ & $0.94(3)$ \\
\hline $\mathrm{C}(2)-\mathrm{O}(3)$ & $1.413(3)$ & $\mathrm{O}(3)-\mathrm{H}(3)$ & $0.84(4)$ \\
\hline $\mathrm{C}(2)-\mathrm{C}(2)^{\# 1}$ & $1.542(4)$ & $\mathrm{O}(6)-\mathrm{H}(6)$ & $0.73(3)$ \\
\hline $\mathrm{C}(2)-\mathrm{H}(2)$ & $0.92(3)$ & $\mathrm{O}(7)-\mathrm{H}(71)$ & $0.74(4)$ \\
\hline $\mathrm{C}(3)-\mathrm{O}(4)$ & $1.252(3)$ & $\mathrm{O}(7)-\mathrm{H}(72)$ & $0.67(8)$ \\
\hline $\mathrm{C}(3)-\mathrm{O}(5)$ & $1.253(3)$ & $\mathrm{O}(8)-\mathrm{H}(81)$ & $0.90(5)$ \\
\hline $\mathrm{C}(3)-\mathrm{C}(4)$ & $1.532(3)$ & $\mathrm{O}(8)-\mathrm{H}(82)$ & $0.77(4)$ \\
\hline $\mathrm{O}(8)-\mathrm{O}(6)$ & $2.621(2)$ & & \\
\hline Moiety & Angle $\left(^{\circ}\right)$ & Moiety & Angle $\left(^{\circ}\right)$ \\
\hline $\mathrm{O}(5)^{\# 3}-\mathrm{Mg}-\mathrm{O}(1)$ & $99.37(7)$ & $\mathrm{O}(4)-\mathrm{Mg}-\mathrm{O}(6)$ & $76.48(7)$ \\
\hline $\mathrm{O}(5)^{\# 3}-\mathrm{Mg}-\mathrm{O}(4)$ & $99.38(8)$ & $\mathrm{O}(7)-\mathrm{Mg}-\mathrm{O}(6)$ & $168.30(8)$ \\
\hline $\mathrm{O}(1)-\mathrm{Mg}-\mathrm{O}(4)$ & $158.63(7)$ & $\mathrm{O}(3)-\mathrm{Mg}-\mathrm{O}(6)$ & $86.85(7)$ \\
\hline $\mathrm{O}(5)^{\# 3}-\mathrm{Mg}-\mathrm{O}(7)$ & $91.72(8)$ & $\mathrm{C}(1)-\mathrm{O}(1)-\mathrm{Mg}$ & $119.22(14)$ \\
\hline $\mathrm{O}(1)-\mathrm{Mg}-\mathrm{O}(7)$ & $96.81(8)$ & $\mathrm{C}(2)-\mathrm{O}(3)-\mathrm{Mg}$ & $117.38(13)$ \\
\hline $\mathrm{O}(4)-\mathrm{Mg}-\mathrm{O}(7)$ & $92.78(8)$ & $\mathrm{Mg}-\mathrm{O}(3)-\mathrm{H}(3)$ & $121(2)$ \\
\hline $\mathrm{O}(5)^{\# 3}-\mathrm{Mg}-\mathrm{O}(3)$ & $175.46(7)$ & $\mathrm{C}(3)-\mathrm{O}(4)-\mathrm{Mg}$ & $119.26(14)$ \\
\hline $\mathrm{O}(1)-\mathrm{Mg}-\mathrm{O}(3)$ & $76.26(7)$ & $\mathrm{C}(3)-\mathrm{O}(5)-\mathrm{Mg}^{\# 4}$ & $132.96(15)$ \\
\hline $\mathrm{O}(4)-\mathrm{Mg}-\mathrm{O}(3)$ & $85.13(7)$ & $\mathrm{C}(4)-\mathrm{O}(6)-\mathrm{Mg}$ & $114.43(13)$ \\
\hline $\mathrm{O}(7)-\mathrm{Mg}-\mathrm{O}(3)$ & $87.61(8)$ & $\mathrm{Mg}-\mathrm{O}(6)-\mathrm{H}(6)$ & $115(2)$ \\
\hline $\mathrm{O}(5)^{\# 3}-\mathrm{Mg}-\mathrm{O}(6)$ & $94.59(7)$ & $\mathrm{Mg}-\mathrm{O}(7)-\mathrm{H}(71)$ & $116(3)$ \\
\hline $\mathrm{O}(1)-\mathrm{Mg}-\mathrm{O}(6)$ & $91.88(7)$ & $\mathrm{Mg}-\mathrm{O}(7)-\mathrm{H}(72)$ & $98(8)$ \\
\hline \multicolumn{4}{|c|}{ Organic } \\
\hline Moiety & Angle $\left(^{\circ}\right)$ & Moiety & Angle $\left(^{\circ}\right)$ \\
\hline $\mathrm{O}(1)-\mathrm{C}(1)-\mathrm{O}(2)$ & $125.3(2)$ & $\mathrm{O}(4)-\mathrm{C}(3)-\mathrm{C}(4)$ & $117.84(19)$ \\
\hline $\mathrm{O}(1)-\mathrm{C}(1)-\mathrm{C}(2)$ & $118.92(19)$ & $\mathrm{O}(5)-\mathrm{C}(3)-\mathrm{C}(4)$ & 116.01(19) \\
\hline $\mathrm{O}(2)-\mathrm{C}(1)-\mathrm{C}(2)$ & 115.81(19) & $\mathrm{O}(6)-\mathrm{C}(4)-\mathrm{C}(3)$ & $108.78(17)$ \\
\hline $\mathrm{O}(3)-\mathrm{C}(2)-\mathrm{C}(1)$ & $107.95(16)$ & $\mathrm{O}(6)-\mathrm{C}(4)-\mathrm{C}(4)^{\# 2}$ & $111.1(2)$ \\
\hline $\mathrm{O}(3)-\mathrm{C}(2)-\mathrm{C}(2)^{\# 1}$ & $110.72(13)$ & $\mathrm{C}(3)-\mathrm{C}(4)-\mathrm{C}(4)^{\# 2}$ & $114.61(13)$ \\
\hline $\mathrm{C}(1)-\mathrm{C}(2)-\mathrm{C}(2)^{\# 1}$ & $110.9(2)$ & $\mathrm{O}(6)-\mathrm{C}(4)-\mathrm{H}(4)$ & $109.9(14)$ \\
\hline $\mathrm{O}(3)-\mathrm{C}(2)-\mathrm{H}(2)$ & 107.9(16) & $\mathrm{C}(3)-\mathrm{C}(4)-\mathrm{H}(4)$ & $106.6(14)$ \\
\hline $\mathrm{C}(1)-\mathrm{C}(2)-\mathrm{H}(2)$ & $110.1(16)$ & $\mathrm{C}(4)^{\# 2}-\mathrm{C}(4)-\mathrm{H}(4)$ & $105.7(14)$ \\
\hline $\mathrm{C}(2)^{\# 1}-\mathrm{C}(2)-\mathrm{H}(2)$ & $109.2(16)$ & $\mathrm{C}(2)-\mathrm{O}(3)-\mathrm{H}(3)$ & $114(2)$ \\
\hline $\mathrm{O}(4)-\mathrm{C}(3)-\mathrm{O}(5)$ & $126.1(2)$ & $\mathrm{C}(4)-\mathrm{O}(6)-\mathrm{H}(6)$ & $105(2)$ \\
\hline \multicolumn{4}{|c|}{ Water } \\
\hline
\end{tabular}


Moiety $\quad$ Angle $\left({ }^{\circ}\right) \quad$ Moiety $\quad$ Angle $\left({ }^{\circ}\right)$

$\mathrm{H}(71)-\mathrm{O}(7)-\mathrm{H}(72) \quad 127(7) \quad \mathrm{H}(81)-\mathrm{O}(8)-\mathrm{H}(82) \quad 114(4)$

Symmetry transformations used to generate equivalent atoms:

$\# 1-\mathrm{x}+2, \mathrm{y},-\mathrm{z}+1 / 2 \quad \# 2-\mathrm{x}+1, \mathrm{y},-\mathrm{z}+1 / 2 \quad \# 3 \mathrm{x},-\mathrm{y}+1, \mathrm{z}-1 / 2 \quad \# 4 \mathrm{x},-\mathrm{y}+1, \mathrm{z}+1 / 2$ 
Table S5. Atomic coordinates $\left(\times 10^{4}\right)$ and equivalent isotropic displacement parameters $\left(\AA^{2} \times 10^{3}\right)$ for $\left[\mathrm{Mg}\left(\mathrm{D}-\mathrm{C}_{4} \mathrm{H}_{4} \mathrm{O}_{6}\right)\left(\mathrm{H}_{2} \mathbf{O}\right)\right] \cdot \mathbf{1 . 5} \mathbf{H}_{2} \mathbf{O}, \mathbf{3}$.

\begin{tabular}{lllll}
\hline Atom & $\mathrm{x}$ & $\mathrm{y}$ & $\mathrm{z}$ & $\mathrm{U}(\mathrm{eq})$ \\
\hline $\mathrm{C}(1)$ & $447(2)$ & $4090(1)$ & $9933(1)$ & $22(1)$ \\
$\mathrm{C}(2)$ & $2329(2)$ & $4372(1)$ & $9835(1)$ & $21(1)$ \\
$\mathrm{C}(3)$ & $2579(2)$ & $5751(1)$ & $9830(1)$ & $21(1)$ \\
$\mathrm{C}(4)$ & $4458(2)$ & $6047(1)$ & $9914(1)$ & $22(1)$ \\
$\mathrm{C}(5)$ & $3021(2)$ & $3211(1)$ & $7298(1)$ & $21(1)$ \\
$\mathrm{C}(6)$ & $1897(2)$ & $4337(1)$ & $7258(1)$ & $19(1)$ \\
$\mathrm{C}(7)$ & $2988(2)$ & $5480(1)$ & $7243(1)$ & $19(1)$ \\
$\mathrm{C}(8)$ & $1907(2)$ & $6622(1)$ & $7222(1)$ & $21(1)$ \\
$\mathrm{Mg}(1)$ & $1038(1)$ & $2843(1)$ & $8600(1)$ & $20(1)$ \\
$\mathrm{Mg}(2)$ & $3833(1)$ & $7128(1)$ & $8517(1)$ & $21(1)$ \\
$\mathrm{O}(1)$ & $-332(2)$ & $3600(1)$ & $9414(1)$ & $27(1)$ \\
$\mathrm{O}(2)$ & $-182(2)$ & $4381(1)$ & $10531(1)$ & $32(1)$ \\
$\mathrm{O}(3)$ & $2877(2)$ & $3838(1)$ & $9176(1)$ & $25(1)$ \\
$\mathrm{O}(4)$ & $2003(2)$ & $6274(1)$ & $9174(1)$ & $26(1)$ \\
$\mathrm{O}(5)$ & $5191(2)$ & $6567(1)$ & $9395(1)$ & $28(1)$ \\
$\mathrm{O}(6)$ & $5116(2)$ & $5746(1)$ & $10505(1)$ & $32(1)$ \\
$\mathrm{O}(7)$ & $2727(2)$ & $2470(1)$ & $7802(1)$ & $28(1)$ \\
$\mathrm{O}(8)$ & $4138(2)$ & $3112(1)$ & $6828(1)$ & $30(1)$ \\
$\mathrm{O}(9)$ & $752(2)$ & $4324(1)$ & $7862(1)$ & $23(1)$ \\
$\mathrm{O}(10)$ & $4087(2)$ & $5550(1)$ & $7864(1)$ & $23(1)$ \\
$\mathrm{O}(11)$ & $857(2)$ & $6702(1)$ & $6715(1)$ & $31(1)$ \\
$\mathrm{O}(12)$ & $2151(2)$ & $7397(1)$ & $7703(1)$ & $32(1)$ \\
$\mathrm{O}(13)$ & $1718(2)$ & $1429(1)$ & $9226(1)$ & $41(1)$ \\
$\mathrm{O}(14)$ & $3070(2)$ & $8740(1)$ & $8925(1)$ & $33(1)$ \\
$\mathrm{O}(15)$ & $6214(2)$ & $3663(3)$ & $8947(1)$ & $34(1)$ \\
$\mathrm{O}(16)$ & $7446(2)$ & $5006(1)$ & $7461(1)$ & $70(1)$ \\
$\mathrm{O}(17)$ & $8649(2)$ & $6317(2)$ & $9022(2)$ & \\
\hline & & & & \\
\hline
\end{tabular}


Table S6. Bond lengths $[\AA]$ and bond angles $\left[{ }^{\circ}\right]$ for $\left[\mathrm{Mg}\left(\mathrm{D}-\mathrm{C}_{4} \mathrm{H}_{4} \mathrm{O}_{6}\right)\left(\mathrm{H}_{2} \mathrm{O}\right)\right] \cdot \mathbf{1 . 5} \mathrm{H}_{2} \mathbf{O}, \mathbf{3}$.

\begin{tabular}{|c|c|c|c|}
\hline Moiety & Distance $(\AA)$ & Moiety & Distance $(\AA)$ \\
\hline $\operatorname{Mg}(1)-\mathrm{O}(13)$ & $2.0210(15)$ & $\mathrm{Mg}(2)-\mathrm{O}(12)$ & $2.0217(14)$ \\
\hline $\operatorname{Mg}(1)-O(7)$ & $2.0243(14)$ & $\operatorname{Mg}(2)-\mathrm{O}(5)$ & $2.0313(14)$ \\
\hline $\mathrm{Mg}(1)-\mathrm{O}(1)$ & $2.0265(13)$ & $\mathrm{Mg}(2)-\mathrm{O}(14)$ & $2.0351(14)$ \\
\hline $\operatorname{Mg}(1)-O(11)^{\# 1}$ & $2.0481(14)$ & $\operatorname{Mg}(2)-O(8)^{\# 2}$ & $2.0435(14)$ \\
\hline $\mathrm{Mg}(1)-\mathrm{O}(3)$ & $2.1116(14)$ & $\mathrm{Mg}(2)-\mathrm{O}(4)$ & $2.1090(14)$ \\
\hline $\operatorname{Mg}(1)-\mathrm{O}(9)$ & $2.1434(13)$ & $\operatorname{Mg}(2)-\mathrm{O}(10)$ & $2.1356(13)$ \\
\hline \multicolumn{4}{|c|}{ Organic and Water } \\
\hline Moiety & Distance $(\AA)$ & Moiety & Distance $(\AA)$ \\
\hline $\mathrm{C}(1)-\mathrm{O}(2)$ & $1.246(2)$ & $\mathrm{C}(5)-\mathrm{O}(8)$ & $1.240(2)$ \\
\hline $\mathrm{C}(1)-\mathrm{O}(1)$ & $1.258(2)$ & $\mathrm{C}(5)-\mathrm{O}(7)$ & $1.259(2)$ \\
\hline $\mathrm{C}(1)-\mathrm{C}(2)$ & $1.533(2)$ & $\mathrm{C}(5)-\mathrm{C}(6)$ & $1.538(2)$ \\
\hline $\mathrm{C}(2)-\mathrm{O}(3)$ & $1.413(2)$ & $\mathrm{C}(6)-\mathrm{O}(9)$ & $1.4293(19)$ \\
\hline $\mathrm{C}(2)-\mathrm{C}(3)$ & $1.548(2)$ & $\mathrm{C}(6)-\mathrm{C}(7)$ & $1.538(2)$ \\
\hline $\mathrm{C}(2)-\mathrm{H}(21)$ & $0.93(2)$ & $\mathrm{C}(6)-\mathrm{H}(61)$ & $0.90(2)$ \\
\hline $\mathrm{C}(3)-\mathrm{O}(4)$ & $1.411(2)$ & $\mathrm{C}(7)-\mathrm{O}(10)$ & $1.4337(19)$ \\
\hline $\mathrm{C}(3)-\mathrm{C}(4)$ & $1.530(2)$ & $\mathrm{C}(7)-\mathrm{C}(8)$ & $1.533(2)$ \\
\hline $\mathrm{C}(3)-\mathrm{H}(31)$ & $0.94(2)$ & $\mathrm{C}(7)-\mathrm{H}(71)$ & $0.93(2)$ \\
\hline $\mathrm{C}(4)-\mathrm{O}(6)$ & $1.247(2)$ & $\mathrm{C}(8)-\mathrm{O}(12)$ & $1.248(2)$ \\
\hline $\mathrm{C}(4)-\mathrm{O}(5)$ & $1.255(2)$ & $\mathrm{C}(8)-\mathrm{O}(11)$ & $1.249(2)$ \\
\hline $\mathrm{O}(3)-\mathrm{H}(3)$ & $0.806(10)$ & $\mathrm{O}(14)-\mathrm{H}(142)$ & $0.766(10)$ \\
\hline $\mathrm{O}(4)-\mathrm{H}(4)$ & $0.807(10)$ & $\mathrm{O}(15)-\mathrm{H}(151)$ & $0.743(10)$ \\
\hline $\mathrm{O}(9)-\mathrm{H}(9)$ & $0.789(10)$ & $\mathrm{O}(15)-\mathrm{H}(152)$ & $0.747(10)$ \\
\hline $\mathrm{O}(10)-\mathrm{H}(10)$ & $0.807(10)$ & $\mathrm{O}(16)-\mathrm{H}(161)$ & $0.759(10)$ \\
\hline $\mathrm{O}(13)-\mathrm{H}(131)$ & $0.750(9)$ & $\mathrm{O}(16)-\mathrm{H}(162)$ & $0.769(10)$ \\
\hline $\mathrm{O}(13)-\mathrm{H}(132)$ & $0.749(9)$ & $\mathrm{O}(17)-\mathrm{H}(171)$ & $0.752(10)$ \\
\hline $\mathrm{O}(14)-\mathrm{H}(141)$ & $0.772(10)$ & $\mathrm{O}(17)-\mathrm{H}(172)$ & $0.750(10)$ \\
\hline Moiety & Angle $\left(^{\circ}\right)$ & Moiety & Angle $\left(^{\circ}\right)$ \\
\hline $\mathrm{O}(2)-\mathrm{C}(1)-\mathrm{Mg}(1)$ & $161.07(12)$ & $\mathrm{O}(5)-\mathrm{Mg}(2)-\mathrm{O}(8)^{\# 2}$ & $89.65(6)$ \\
\hline $\mathrm{O}(1)-\mathrm{C}(1)-\mathrm{Mg}(1)$ & $38.79(8)$ & $\mathrm{O}(14)-\mathrm{Mg}(2)-\mathrm{O}(8)^{\# 2}$ & $82.77(6)$ \\
\hline $\mathrm{C}(2)-\mathrm{C}(1)-\mathrm{Mg}(1)$ & $80.78(9)$ & $\mathrm{O}(12)-\mathrm{Mg}(2)-\mathrm{O}(4)$ & $92.00(6)$ \\
\hline $\mathrm{O}(6)-\mathrm{C}(4)-\mathrm{Mg}(2)$ & $163.39(12)$ & $\mathrm{O}(5)-\mathrm{Mg}(2)-\mathrm{O}(4)$ & $76.91(5)$ \\
\hline $\mathrm{O}(5)-\mathrm{C}(4)-\mathrm{Mg}(2)$ & $37.91(8)$ & $\mathrm{O}(14)-\mathrm{Mg}(2)-\mathrm{O}(4)$ & $89.11(6)$ \\
\hline $\mathrm{C}(3)-\mathrm{C}(4)-\mathrm{Mg}(2)$ & $80.41(9)$ & $\mathrm{O}(8)^{\# 2}-\mathrm{Mg}(2)-\mathrm{O}(4)$ & $163.22(6)$ \\
\hline $\mathrm{O}(13)-\mathrm{Mg}(1)-\mathrm{O}(7)$ & $94.23(6)$ & $\mathrm{O}(12)-\mathrm{Mg}(2)-\mathrm{O}(10)$ & $76.72(5)$ \\
\hline $\mathrm{O}(13)-\mathrm{Mg}(1)-\mathrm{O}(1)$ & $92.81(7)$ & $\mathrm{O}(5)-\mathrm{Mg}(2)-\mathrm{O}(10)$ & $98.05(5)$ \\
\hline $\mathrm{O}(7)-\mathrm{Mg}(1)-\mathrm{O}(1)$ & $165.89(6)$ & $\mathrm{O}(14)-\mathrm{Mg}(2)-\mathrm{O}(10)$ & $163.53(6)$ \\
\hline $\mathrm{O}(13)-\mathrm{Mg}(1)-\mathrm{O}(11)^{\# 1}$ & $82.63(6)$ & $\mathrm{O}(8)^{\# 2}-\mathrm{Mg}(2)-\mathrm{O}(10)$ & $101.19(5)$ \\
\hline $\mathrm{O}(7)-\mathrm{Mg}(1)-\mathrm{O}(11)^{\# 1}$ & $98.79(6)$ & $\mathrm{O}(4)-\mathrm{Mg}(2)-\mathrm{O}(10)$ & $90.74(5)$ \\
\hline $\mathrm{O}(1)-\mathrm{Mg}(1)-\mathrm{O}(11)^{\# 1}$ & $94.23(6)$ & $\mathrm{O}(12)-\mathrm{Mg}(2)-\mathrm{C}(4)$ & $146.41(6)$ \\
\hline $\mathrm{O}(13)-\mathrm{Mg}(1)-\mathrm{O}(3)$ & $86.60(6)$ & $\mathrm{O}(5)-\mathrm{Mg}(2)-\mathrm{C}(4)$ & $22.31(5)$ \\
\hline $\mathrm{O}(7)-\mathrm{Mg}(1)-\mathrm{O}(3)$ & $90.77(6)$ & $\mathrm{O}(14)-\mathrm{Mg}(2)-\mathrm{C}(4)$ & $95.39(6)$ \\
\hline
\end{tabular}




\begin{tabular}{|c|c|c|c|}
\hline $\mathrm{O}(1)-\mathrm{Mg}(1)-\mathrm{O}(3)$ & $77.45(5)$ & $\mathrm{O}(8)^{\# 2}-\mathrm{Mg}(2)-\mathrm{C}(4)$ & $111.40(6)$ \\
\hline $\mathrm{O}(11)^{\# 1}-\mathrm{Mg}(1)-\mathrm{O}(3)$ & $166.07(6)$ & $\mathrm{O}(4)-\mathrm{Mg}(2)-\mathrm{C}(4)$ & $54.62(5)$ \\
\hline $\mathrm{O}(13)-\mathrm{Mg}(1)-\mathrm{O}(9)$ & $170.05(6)$ & $\mathrm{O}(10)-\mathrm{Mg}(2)-\mathrm{C}(4)$ & $97.94(5)$ \\
\hline $\mathrm{O}(7)-\mathrm{Mg}(1)-\mathrm{O}(9)$ & $76.83(5)$ & $\mathrm{C}(1)-\mathrm{O}(1)-\mathrm{Mg}(1)$ & $118.32(11)$ \\
\hline $\mathrm{O}(1)-\mathrm{Mg}(1)-\mathrm{O}(9)$ & $95.03(5)$ & $\mathrm{C}(2)-\mathrm{O}(3)-\mathrm{Mg}(1)$ & $115.87(10)$ \\
\hline $\mathrm{O}(11)^{\# 1}-\mathrm{Mg}(1)-\mathrm{O}(9)$ & $102.86(5)$ & $\mathrm{Mg}(1)-\mathrm{O}(3)-\mathrm{H}(3)$ & $130.5(17)$ \\
\hline $\mathrm{O}(3)-\mathrm{Mg}(1)-\mathrm{O}(9)$ & $89.12(5)$ & $\mathrm{C}(3)-\mathrm{O}(4)-\mathrm{Mg}(2)$ & $116.70(10)$ \\
\hline $\mathrm{O}(13)-\mathrm{Mg}(1)-\mathrm{C}(1)$ & $86.37(6)$ & $\mathrm{Mg}(2)-\mathrm{O}(4)-\mathrm{H}(4)$ & $126.1(17)$ \\
\hline $\mathrm{O}(7)-\mathrm{Mg}(1)-\mathrm{C}(1)$ & $145.87(6)$ & $\mathrm{C}(4)-\mathrm{O}(5)-\mathrm{Mg}(2)$ & $119.78(11)$ \\
\hline $\mathrm{O}(1)-\mathrm{Mg}(1)-\mathrm{C}(1)$ & $22.89(5)$ & $\mathrm{C}(5)-\mathrm{O}(7)-\mathrm{Mg}(1)$ & $121.08(11)$ \\
\hline $\mathrm{O}(11)^{\# 1}-\mathrm{Mg}(1)-\mathrm{C}(1)$ & $115.08(6)$ & $\mathrm{C}(5)-\mathrm{O}(8)-\mathrm{Mg}(2)^{\# 3}$ & $145.35(12)$ \\
\hline $\mathrm{O}(3)-\mathrm{Mg}(1)-\mathrm{C}(1)$ & $55.15(5)$ & $\mathrm{C}(6)-\mathrm{O}(9)-\mathrm{Mg}(1)$ & $115.36(9)$ \\
\hline $\mathrm{O}(9)-\mathrm{Mg}(1)-\mathrm{C}(1)$ & $98.54(5)$ & $\mathrm{Mg}(1)-\mathrm{O}(9)-\mathrm{H}(9)$ & $124.7(19)$ \\
\hline $\mathrm{O}(12)-\mathrm{Mg}(2)-\mathrm{O}(5)$ & $167.79(6)$ & $\mathrm{C}(7)-\mathrm{O}(10)-\mathrm{Mg}(2)$ & $115.59(9)$ \\
\hline $\mathrm{O}(12)-\mathrm{Mg}(2)-\mathrm{O}(14)$ & $86.82(6)$ & $\mathrm{Mg}(2)-\mathrm{O}(10)-\mathrm{H}(10)$ & $122.1(17)$ \\
\hline $\mathrm{O}(5)-\mathrm{Mg}(2)-\mathrm{O}(14)$ & $97.96(6)$ & $\mathrm{C}(8)-\mathrm{O}(11)-\mathrm{Mg}(1)^{\# 4}$ & $137.77(11)$ \\
\hline $\mathrm{O}(12)-\mathrm{Mg}(2)-\mathrm{O}(8)^{\# 2}$ & $102.13(6)$ & $\mathrm{C}(8)-\mathrm{O}(12)-\mathrm{Mg}(2)$ & $121.35(11)$ \\
\hline $\mathrm{Mg}(1)-\mathrm{O}(13)-\mathrm{H}(131)$ & $120(2)$ & $\mathrm{Mg}(2)-\mathrm{O}(14)-\mathrm{H}(141)$ & $112(2)$ \\
\hline $\mathrm{Mg}(1)-\mathrm{O}(13)-\mathrm{H}(132)$ & $119(2)$ & $\mathrm{Mg}(2)-\mathrm{O}(14)-\mathrm{H}(142)$ & $117(3)$ \\
\hline \multicolumn{4}{|c|}{ Organic } \\
\hline Moiety & Angle $\left(^{\circ}\right)$ & Moiety & Angle $\left(^{\circ}\right)$ \\
\hline $\mathrm{O}(2)-\mathrm{C}(1)-\mathrm{O}(1)$ & $125.53(16)$ & $\mathrm{O}(7)-\mathrm{C}(5)-\mathrm{C}(6)$ & $117.52(14)$ \\
\hline $\mathrm{O}(2)-\mathrm{C}(1)-\mathrm{C}(2)$ & $116.02(15)$ & $\mathrm{O}(9)-\mathrm{C}(6)-\mathrm{C}(5)$ & $108.77(12)$ \\
\hline $\mathrm{O}(1)-\mathrm{C}(1)-\mathrm{C}(2)$ & $118.45(15)$ & $\mathrm{O}(9)-\mathrm{C}(6)-\mathrm{C}(7)$ & $112.20(12)$ \\
\hline $\mathrm{O}(3)-\mathrm{C}(2)-\mathrm{C}(1)$ & $108.17(13)$ & $C(5)-C(6)-C(7)$ & $110.51(14)$ \\
\hline $\mathrm{O}(3)-\mathrm{C}(2)-\mathrm{C}(3)$ & 111.91(12) & $\mathrm{O}(9)-\mathrm{C}(6)-\mathrm{H}(61)$ & $108.2(13)$ \\
\hline $\mathrm{C}(1)-\mathrm{C}(2)-\mathrm{C}(3)$ & $109.13(14)$ & $\mathrm{C}(5)-\mathrm{C}(6)-\mathrm{H}(61)$ & $109.0(13)$ \\
\hline $\mathrm{O}(3)-\mathrm{C}(2)-\mathrm{H}(21)$ & $110.8(13)$ & $\mathrm{C}(7)-\mathrm{C}(6)-\mathrm{H}(61)$ & $108.1(13)$ \\
\hline $\mathrm{C}(1)-\mathrm{C}(2)-\mathrm{H}(21)$ & $105.0(13)$ & $\mathrm{O}(10)-\mathrm{C}(7)-\mathrm{C}(8)$ & $108.28(12)$ \\
\hline $\mathrm{C}(3)-\mathrm{C}(2)-\mathrm{H}(21)$ & $111.5(13)$ & $\mathrm{O}(10)-\mathrm{C}(7)-\mathrm{C}(6)$ & $111.76(12)$ \\
\hline $\mathrm{O}(4)-\mathrm{C}(3)-\mathrm{C}(4)$ & $108.12(13)$ & $\mathrm{C}(8)-\mathrm{C}(7)-\mathrm{C}(6)$ & $111.93(14)$ \\
\hline $\mathrm{O}(4)-\mathrm{C}(3)-\mathrm{C}(2)$ & 111.91(12) & $\mathrm{O}(10)-\mathrm{C}(7)-\mathrm{H}(71)$ & $106.3(14)$ \\
\hline$C(4)-C(3)-C(2)$ & $109.69(14)$ & $\mathrm{C}(8)-\mathrm{C}(7)-\mathrm{H}(71)$ & $109.5(13)$ \\
\hline $\mathrm{O}(4)-\mathrm{C}(3)-\mathrm{H}(31)$ & 111.7(13) & $\mathrm{C}(6)-\mathrm{C}(7)-\mathrm{H}(71)$ & $108.9(13)$ \\
\hline $\mathrm{C}(4)-\mathrm{C}(3)-\mathrm{H}(31)$ & $102.4(13)$ & $\mathrm{O}(12)-\mathrm{C}(8)-\mathrm{O}(11)$ & $125.32(15)$ \\
\hline $\mathrm{C}(2)-\mathrm{C}(3)-\mathrm{H}(31)$ & $112.5(13)$ & $\mathrm{O}(12)-\mathrm{C}(8)-\mathrm{C}(7)$ & $117.99(14)$ \\
\hline $\mathrm{O}(6)-\mathrm{C}(4)-\mathrm{O}(5)$ & $126.04(16)$ & $\mathrm{O}(11)-\mathrm{C}(8)-\mathrm{C}(7)$ & $116.68(14)$ \\
\hline $\mathrm{O}(6)-\mathrm{C}(4)-\mathrm{C}(3)$ & $115.81(15)$ & $\mathrm{C}(2)-\mathrm{O}(3)-\mathrm{H}(3)$ & $109.4(17)$ \\
\hline $\mathrm{O}(5)-\mathrm{C}(4)-\mathrm{C}(3)$ & $118.16(15)$ & $\mathrm{C}(3)-\mathrm{O}(4)-\mathrm{H}(4)$ & $114.3(17)$ \\
\hline $\mathrm{O}(8)-\mathrm{C}(5)-\mathrm{O}(7)$ & $125.64(15)$ & $\mathrm{C}(6)-\mathrm{O}(9)-\mathrm{H}(9)$ & $108.2(18)$ \\
\hline $\mathrm{O}(8)-\mathrm{C}(5)-\mathrm{C}(6)$ & $116.84(14)$ & $\mathrm{C}(7)-\mathrm{O}(10)-\mathrm{H}(10)$ & $108.6(16)$ \\
\hline \multicolumn{4}{|c|}{ Water } \\
\hline Moiety & Angle $\left(^{\circ}\right)$ & Moiety & Angle $\left(^{\circ}\right)$ \\
\hline $\mathrm{H}(131)-\mathrm{O}(13)-\mathrm{H}(132)$ & $120.0(19)$ & $\mathrm{H}(161)-\mathrm{O}(16)-\mathrm{H}(162)$ & $114.4(19)$ \\
\hline $\mathrm{H}(141)-\mathrm{O}(14)-\mathrm{H}(142)$ & $113.8(18)$ & $\mathrm{H}(171)-\mathrm{O}(17)-\mathrm{H}(172)$ & $120(2)$ \\
\hline $\mathrm{H}(151)-\mathrm{O}(15)-\mathrm{H}(152)$ & $122(2)$ & & \\
\hline
\end{tabular}


Symmetry transformations used to generate equivalent atoms:

$\# 1-\mathrm{x}, \mathrm{y}-1 / 2,-\mathrm{z}+3 / 2 \quad \# 2-\mathrm{x}+1, \mathrm{y}+1 / 2,-\mathrm{z}+3 / 2 \quad \# 3-\mathrm{x}+1, \mathrm{y}-1 / 2,-\mathrm{z}+3 / 2 \quad \# 4-\mathrm{x}, \mathrm{y}+1 / 2,-\mathrm{z}+3 / 2$ 
Table S7. Atomic coordinates $\left(\mathrm{x}_{10} 0^{4}\right.$ ) and equivalent isotropic displacement parameters $\left(\AA^{2} \times 10^{3}\right)$ for $\left[\mathbf{M g}\left(\mathbf{D}-\mathbf{C}_{4} \mathbf{H}_{4} \mathbf{O}_{6}\right)\right] \cdot \mathbf{H}_{2} \mathbf{O}, 4$.

\begin{tabular}{lllll}
\hline Atom & $\mathrm{x}$ & $\mathrm{y}$ & $\mathrm{z}$ & $\mathrm{U}(\mathrm{eq})$ \\
\hline $\mathrm{C}(1)$ & $4558(3)$ & $2478(2)$ & $5703(2)$ & $20(1)$ \\
$\mathrm{C}(2)$ & $5609(3)$ & $2711(2)$ & $6924(2)$ & $18(1)$ \\
$\mathrm{Mg}$ & $1723(1)$ & 0 & 5000 & $19(1)$ \\
$\mathrm{O}(1)$ & $3545(2)$ & $1390(2)$ & $5702(2)$ & $32(1)$ \\
$\mathrm{O}(2)$ & $4838(2)$ & $3362(2)$ & $4799(1)$ & $28(1)$ \\
$\mathrm{O}(3)$ & $6615(2)$ & $4064(2)$ & $6842(2)$ & $21(1)$ \\
$\mathrm{O}(4)$ & 5000 & $1841(3)$ & 2500 & $31(1)$ \\
\hline
\end{tabular}


Table S8. Bond lengths $[\AA]$ and Bond angles $\left[^{\circ}\right]$ for $\left[\mathbf{M g}\left(\mathrm{D}-\mathrm{C}_{4} \mathbf{H}_{4} \mathbf{O}_{6}\right)\right] \cdot \mathbf{H}_{2} \mathbf{O}, \mathbf{4}$.

\begin{tabular}{|c|c|c|c|}
\hline Moiety & Distance $(\AA)$ & Moiety & Distance $(\AA)$ \\
\hline $\mathrm{Mg}-\mathrm{O}(1)$ & $1.9915(16)$ & $\mathrm{Mg}-\mathrm{O}(2)^{\# 5}$ & $2.0450(17)$ \\
\hline $\mathrm{Mg}-\mathrm{O}(1)^{\# 3}$ & $1.9915(16)$ & $\mathrm{Mg}-\mathrm{O}(3)^{\# 4}$ & $2.1189(16)$ \\
\hline $\mathrm{Mg}-\mathrm{O}(2)^{\# 4}$ & $2.0450(17)$ & $\mathrm{Mg}-\mathrm{O}(3)^{\# 5}$ & $2.1189(16)$ \\
\hline \multicolumn{4}{|c|}{ Organic and Water } \\
\hline Moiety & Distance $(\AA)$ & Moiety & Distance $(\AA)$ \\
\hline $\mathrm{C}(1)-\mathrm{O}(1)$ & $1.232(3)$ & $\mathrm{C}(2)-\mathrm{O}(3)$ & $1.423(2)$ \\
\hline $\mathrm{C}(1)-\mathrm{O}(2)$ & $1.255(3)$ & $C(2)-C(2)^{\# 2}$ & $1.526(4)$ \\
\hline $\mathrm{C}(1)-\mathrm{C}(2)$ & $1.530(3)$ & $\mathrm{C}(2)-\mathrm{H}(1)$ & $0.93(2)$ \\
\hline $\mathrm{O}(3)-\mathrm{H}(2)$ & $0.78(3)$ & $\mathrm{O}(4)-\mathrm{H}(3)$ & $0.83(3)$ \\
\hline Moiety & Angle $\left(^{\circ}\right)$ & Moiety & Angle $\left(^{\circ}\right)$ \\
\hline $\mathrm{O}(1)-\mathrm{C}(1)-\mathrm{Mg}^{\# 1}$ & $164.76(16)$ & $\mathrm{O}(1)-\mathrm{Mg}-\mathrm{C}(1)^{\# 4}$ & $141.27(6)$ \\
\hline $\mathrm{O}(2)-\mathrm{C}(1)-\mathrm{Mg}^{\# 1}$ & $38.65(10)$ & $\mathrm{O}(1)^{\# 3}-\mathrm{Mg}-\mathrm{C}(1)^{\# 4}$ & $90.43(6)$ \\
\hline $\mathrm{C}(2)-\mathrm{C}(1)-\mathrm{Mg}^{\# 1}$ & $79.50(11)$ & $\mathrm{O}(2)^{\# 4}-\mathrm{Mg}-\mathrm{C}(1)^{\# 4}$ & $22.53(6)$ \\
\hline $\mathrm{O}(1)-\mathrm{Mg}-\mathrm{O}(1)^{\# 3}$ & $92.57(11)$ & $\mathrm{O}(2)^{\# 5}-\mathrm{Mg}-\mathrm{C}(1)^{\# 4}$ & $97.48(7)$ \\
\hline $\mathrm{O}(1)-\mathrm{Mg}-\mathrm{O}(2)^{\# 4}$ & $163.69(6)$ & $\mathrm{O}(3)^{\# 4}-\mathrm{Mg}-\mathrm{C}(1)^{\# 4}$ & $55.55(6)$ \\
\hline $\mathrm{O}(1)^{\# 3}-\mathrm{Mg}-\mathrm{O}(2)^{\# 4}$ & $90.13(7)$ & $\mathrm{O}(3)^{\# 5}-\mathrm{Mg}-\mathrm{C}(1)^{\# 4}$ & $121.45(6)$ \\
\hline $\mathrm{O}(1)-\mathrm{Mg}-\mathrm{O}(2) \# 5$ & $90.13(7)$ & $\mathrm{O}(1)-\mathrm{Mg}-\mathrm{C}(1)^{\# 5}$ & $90.43(6)$ \\
\hline $\mathrm{O}(1)^{\# 3}-\mathrm{Mg}-\mathrm{O}(2)^{\# 5}$ & $163.69(6)$ & $\mathrm{O}(1)^{\# 3}-\mathrm{Mg}-\mathrm{C}(1)^{\# 5}$ & $141.27(6)$ \\
\hline $\mathrm{O}(2)^{\# 4}-\mathrm{Mg}-\mathrm{O}(2)^{\# 5}$ & $91.77(10)$ & $\mathrm{O}(2)^{\# 4}-\mathrm{Mg}-\mathrm{C}(1)^{\# 5}$ & $97.48(7)$ \\
\hline $\mathrm{O}(1)-\mathrm{Mg}-\mathrm{O}(3)^{\# 4}$ & $85.79(6)$ & $\mathrm{O}(2)^{\# 5}-\mathrm{Mg}-\mathrm{C}(1)^{\# 5}$ & $22.53(6)$ \\
\hline $\mathrm{O}(1)^{\# 3}-\mathrm{Mg}-\mathrm{O}(3)^{\# 4}$ & $97.27(7)$ & $\mathrm{O}(3)^{\# 4}-\mathrm{Mg}-\mathrm{C}(1)^{\# 5}$ & $121.45(6)$ \\
\hline $\mathrm{O}(2)^{\# 4}-\mathrm{Mg}-\mathrm{O}(3)^{\# 4}$ & $77.91(6)$ & $\mathrm{O}(3)^{\# 5}-\mathrm{Mg}-\mathrm{C}(1)^{\# 5}$ & $55.55(6)$ \\
\hline $\mathrm{O}(2)^{\# 5}-\mathrm{Mg}-\mathrm{O}(3)^{\# 4}$ & $98.97(6)$ & $\mathrm{C}(1)^{\# 4}-\mathrm{Mg}-\mathrm{C}(1)^{\# 5}$ & $110.51(9)$ \\
\hline $\mathrm{O}(1)-\mathrm{Mg}-\mathrm{O}(3)^{\# 5}$ & $97.27(7)$ & $\mathrm{C}(1)-\mathrm{O}(1)-\mathrm{Mg}$ & $156.20(15)$ \\
\hline $\mathrm{O}(1)^{\# 3}-\mathrm{Mg}-\mathrm{O}(3)^{\# 5}$ & $85.79(6)$ & $\mathrm{C}(1)-\mathrm{O}(2)-\mathrm{Mg}^{\# 1}$ & $118.82(14)$ \\
\hline $\mathrm{O}(2)^{\# 4}-\mathrm{Mg}-\mathrm{O}(3)^{\# 5}$ & $98.97(6)$ & $\mathrm{C}(2)-\mathrm{O}(3)-\mathrm{Mg}^{\# 1}$ & $114.04(12)$ \\
\hline $\mathrm{O}(2)^{\# 5}-\mathrm{Mg}-\mathrm{O}(3)^{\# 5}$ & $77.91(6)$ & $\mathrm{Mg}^{\# 1}-\mathrm{O}(3)-\mathrm{H}(2)$ & $107.7(17)$ \\
\hline $\mathrm{O}(3)^{\# 4}-\mathrm{Mg}-\mathrm{O}(3)^{\# 5}$ & $175.59(10)$ & & \\
\hline \multicolumn{4}{|c|}{ Organic } \\
\hline Moiety & Angle $\left(^{\circ}\right)$ & Moiety & Angle $\left(^{\circ}\right)$ \\
\hline $\mathrm{C}(2)-\mathrm{O}(3)-\mathrm{H}(2)$ & $112.1(18)$ & $\mathrm{O}(3)-\mathrm{C}(2)-\mathrm{C}(1)$ & 109.82(17) \\
\hline $\mathrm{O}(1)-\mathrm{C}(1)-\mathrm{O}(2)$ & $126.4(2)$ & $\mathrm{C}(2)^{\# 2}-\mathrm{C}(2)-\mathrm{C}(1)$ & 111.1(2) \\
\hline $\mathrm{O}(1)-\mathrm{C}(1)-\mathrm{C}(2)$ & $115.40(18)$ & $\mathrm{O}(3)-\mathrm{C}(2)-\mathrm{H}(1)$ & $110.3(14)$ \\
\hline $\mathrm{O}(2)-\mathrm{C}(1)-\mathrm{C}(2)$ & $118.13(18)$ & $\mathrm{C}(2)^{\# 2}-\mathrm{C}(2)-\mathrm{H}(1)$ & $105.5(14)$ \\
\hline $\mathrm{O}(3)-\mathrm{C}(2)-\mathrm{C}(2)^{\# 2}$ & $111.77(13)$ & $\mathrm{C}(1)-\mathrm{C}(2)-\mathrm{H}(1)$ & $108.2(13)$ \\
\hline
\end{tabular}

Symmetry transformations used to generate equivalent atoms:

$\# 1 \mathrm{x}+1 / 2, \mathrm{y}+1 / 2, \mathrm{z} \quad \# 2-\mathrm{x}+1, \mathrm{y},-\mathrm{z}+3 / 2 \quad \# 3 \mathrm{x},-\mathrm{y},-\mathrm{z}+1 \quad \# 4 \mathrm{x}-1 / 2, \mathrm{y}-1 / 2, \mathrm{z} \quad \# 5 \mathrm{x}-1 / 2,-\mathrm{y}+1 / 2,-$ $\mathrm{z}+1$ 
Table S9: Atomic coordinates (x 10 $)$ and equivalent isotropic displacement parameters $\left(\AA^{2} \times 10^{3}\right)$ for $\left[\mathbf{M g}\left(\mathbf{D}-\mathbf{C}_{4} \mathbf{H}_{4} \mathbf{O}_{6}\right)\right], 5$.

\begin{tabular}{lllll}
\hline Atom & $\mathrm{X}$ & $\mathrm{y}$ & $\mathrm{z}$ & $\mathrm{U}(\mathrm{eq})$ \\
\hline $\mathrm{C}(1)$ & $8076(9)$ & $2774(4)$ & $6419(3)$ & $23(1)$ \\
$\mathrm{C}(2)$ & $10437(9)$ & $2679(4)$ & $5645(3)$ & $23(1)$ \\
$\mathrm{Mg}$ & 10000 & 0 & $7176(1)$ & $23(1)$ \\
$\mathrm{O}(1)$ & $7263(6)$ & $1612(3)$ & $6899(2)$ & $28(1)$ \\
$\mathrm{O}(2)$ & $7001(7)$ & $3993(3)$ & $6514(2)$ & $30(1)$ \\
$\mathrm{O}(3)$ & $11882(7)$ & $1360(3)$ & $5895(2)$ & $25(1)$ \\
\hline
\end{tabular}


Table S10. Bond lengths $[\AA ̊]$ and Bond angles $\left[{ }^{\circ}\right]$ for $\left[\mathbf{M g}\left(\mathbf{D}-\mathbf{C}_{4} \mathbf{H}_{4} \mathbf{O}_{6}\right)\right], \mathbf{5}$.

\begin{tabular}{|c|c|c|c|}
\hline Moiety & Distance $(\AA)$ & Moiety & Distance $(\AA)$ \\
\hline $\mathrm{Mg}-\mathrm{O}(2)^{\# 2}$ & $2.082(3)$ & $\mathrm{Mg}-\mathrm{O}(1)^{\# 4}$ & $2.094(3)$ \\
\hline $\mathrm{Mg}-\mathrm{O}(2)^{\# 3}$ & $2.082(3)$ & $\mathrm{Mg}-\mathrm{O}(3)^{\# 4}$ & $2.199(4)$ \\
\hline $\mathrm{Mg}-\mathrm{O}(1)$ & 2.094(3) & $\mathrm{Mg}-\mathrm{O}(3)$ & $2.199(4)$ \\
\hline $\mathrm{O}(2)-\mathrm{Mg}^{\# 5}$ & $2.082(3)$ & & \\
\hline \multicolumn{4}{|c|}{ Organic } \\
\hline $\mathrm{C}(1)-\mathrm{O}(2)$ & $1.275(5)$ & $\mathrm{C}(2)-\mathrm{O}(3)$ & $1.473(5)$ \\
\hline $\mathrm{C}(1)-\mathrm{O}(1)$ & $1.295(5)$ & $\mathrm{C}(2)-\mathrm{C}(2)^{\# 1}$ & $1.583(7)$ \\
\hline$C(1)-C(2)$ & $1.526(6)$ & $\mathrm{C}(2)-\mathrm{H}(2)$ & $0.84(5)$ \\
\hline $\mathrm{O}(3)-\mathrm{H}(3)$ & $0.79(6)$ & & \\
\hline Moiety & Angle $\left(^{\circ}\right)$ & Moiety & Angle $\left(^{\circ}\right)$ \\
\hline $\mathrm{O}(2)^{\# 2}-\mathrm{Mg}-\mathrm{O}(2)^{\# 3}$ & $84.47(17)$ & $\mathrm{O}(1)^{\# 4}-\mathrm{Mg}-\mathrm{O}(3)^{\# 4}$ & $77.04(12)$ \\
\hline $\mathrm{O}(2)^{\# 2}-\mathrm{Mg}-\mathrm{O}(1)$ & $97.16(11)$ & $\mathrm{O}(2)^{\# 2}-\mathrm{Mg}-\mathrm{O}(3)$ & $91.46(11)$ \\
\hline $\mathrm{O}(2)^{\# 3}-\mathrm{Mg}-\mathrm{O}(1)$ & $96.04(13)$ & $\mathrm{O}(2)^{\# 3}-\mathrm{Mg}-\mathrm{O}(3)$ & $171.51(12)$ \\
\hline $\mathrm{O}(2)^{\# 2}-\mathrm{Mg}-\mathrm{O}(1)^{\# 4}$ & $96.04(13)$ & $\mathrm{O}(1)-\mathrm{Mg}-\mathrm{O}(3)$ & $77.04(12)$ \\
\hline $\mathrm{O}(2)^{\# 3}-\mathrm{Mg}-\mathrm{O}(1)^{\# 4}$ & $97.16(11)$ & $\mathrm{O}(1)^{\# 4}-\mathrm{Mg}-\mathrm{O}(3)$ & $90.67(12)$ \\
\hline $\mathrm{O}(1)-\mathrm{Mg}-\mathrm{O}(1)^{\# 4}$ & $162.14(18)$ & $\mathrm{O}(3)^{\# 4}-\mathrm{Mg}-\mathrm{O}(3)$ & $93.51(16)$ \\
\hline $\mathrm{O}(2)^{\# 2}-\mathrm{Mg}-\mathrm{O}(3)^{\# 4}$ & $171.51(12)$ & $\mathrm{C}(1)-\mathrm{O}(1)-\mathrm{Mg}$ & $116.9(3)$ \\
\hline $\mathrm{O}(2)^{\# 3}-\mathrm{Mg}-\mathrm{O}(3)^{\# 4}$ & $91.46(11)$ & $\mathrm{C}(1)-\mathrm{O}(2)-\mathrm{Mg}^{\# 5}$ & 133.4(2) \\
\hline $\mathrm{O}(1)-\mathrm{Mg}-\mathrm{O}(3)^{\# 4}$ & $90.67(12)$ & $\mathrm{C}(2)-\mathrm{O}(3)-\mathrm{Mg}$ & $113.4(3)$ \\
\hline $\mathrm{Mg}-\mathrm{O}(3)-\mathrm{H}(3)$ & $114(3)$ & & \\
\hline \multicolumn{4}{|c|}{ Organic } \\
\hline Moiety & Angle $\left(^{\circ}\right)$ & Moiety & Angle $\left(^{\circ}\right)$ \\
\hline$\overline{\mathrm{O}(2)-\mathrm{C}(1)-\mathrm{O}(1)}$ & $124.8(4)$ & $\mathrm{C}(1)-\mathrm{C}(2)-\mathrm{C}(2)^{\# 1}$ & $110.1(5)$ \\
\hline $\mathrm{O}(2)-\mathrm{C}(1)-\mathrm{C}(2)$ & $117.0(3)$ & $\mathrm{O}(3)-\mathrm{C}(2)-\mathrm{H}(2)$ & $114(3)$ \\
\hline $\mathrm{O}(1)-\mathrm{C}(1)-\mathrm{C}(2)$ & 118.1(3) & $\mathrm{C}(1)-\mathrm{C}(2)-\mathrm{H}(2)$ & 106(3) \\
\hline $\mathrm{O}(3)-\mathrm{C}(2)-\mathrm{C}(1)$ & 109.6(3) & $\mathrm{C}(2)^{\# 1}-\mathrm{C}(2)-\mathrm{H}(2)$ & $107(3)$ \\
\hline $\mathrm{O}(3)-\mathrm{C}(2)-\mathrm{C}(2)^{\# 1}$ & $109.7(2)$ & $\mathrm{C}(2)-\mathrm{O}(3)-\mathrm{H}(3)$ & $112(4)$ \\
\hline
\end{tabular}

Symmetry transformations used to generate equivalent atoms:

$\# 1-x+2, y,-z+1 \quad \# 2 x+1 / 2,-y+1 / 2,-z+3 / 2 \quad \# 3-x+3 / 2, y-1 / 2,-z+3 / 2 \quad \# 4-x+2,-y, z$

$\# 5-\mathrm{x}+3 / 2, \mathrm{y}+1 / 2,-\mathrm{z}+3 / 2$ 
Table S11: Atomic coordinates (x $10^{4}$ ) and equivalent isotropic displacement parameters $\left(\AA^{2} \times 10^{3}\right)$ for $\left[\mathrm{Mg}\left(\right.\right.$ meso- $\left.\left.\mathrm{C}_{4} \mathrm{H}_{4} \mathrm{O}_{6}\right)\left(\mathrm{H}_{2} \mathrm{O}\right)_{2} \cdot \mathrm{H}_{2} \mathrm{O}\right], 6$.

\begin{tabular}{lllll}
\hline Atom & $\mathrm{x}$ & $\mathrm{y}$ & $\mathrm{z}$ & $\mathrm{U}(\mathrm{eq})$ \\
\hline $\mathrm{C}(1)$ & $2186(3)$ & $6147(2)$ & $6411(1)$ & $24(1)$ \\
$\mathrm{C}(2)$ & $3438(3)$ & $7497(2)$ & $6602(1)$ & $21(1)$ \\
$\mathrm{C}(4)$ & $2877(3)$ & $8630(2)$ & $5921(1)$ & $20(1)$ \\
$\mathrm{C}(5)$ & $493(3)$ & $9265(2)$ & $6057(1)$ & $22(1)$ \\
$\mathrm{Mg}$ & $1530(1)$ & $6506(1)$ & $8342(1)$ & $23(1)$ \\
$\mathrm{O}(1)$ & $1320(3)$ & $5571(1)$ & $7088(1)$ & $27(1)$ \\
$\mathrm{O}(2)$ & $2166(3)$ & $5671(2)$ & $5647(1)$ & $35(1)$ \\
$\mathrm{O}(3)$ & $2952(3)$ & $7906(2)$ & $7495(1)$ & $27(1)$ \\
$\mathrm{O}(4)$ & $4525(3)$ & $9706(1)$ & $6042(1)$ & $25(1)$ \\
$\mathrm{O}(5)$ & $463(2)$ & $10465(1)$ & $6352(1)$ & $29(1)$ \\
$\mathrm{O}(6)$ & $-1239(2)$ & $8554(2)$ & $5872(1)$ & $30(1)$ \\
$\mathrm{O}(7)$ & $2324(3)$ & $7823(2)$ & $9377(1)$ & $38(1)$ \\
$\mathrm{O}(8)$ & $-1773(3)$ & $7091(2)$ & $8246(1)$ & $42(1)$ \\
$\mathrm{O}(9)$ & $2077(3)$ & $336(2)$ & $1243(2)$ & $46(1)$ \\
\hline
\end{tabular}


Table S12. Bond lengths $[\AA]$ and Bond angles $\left[{ }^{\circ}\right]$ for $\left[\mathrm{Mg}\left(\right.\right.$ meso $\left.\left.-\mathrm{C}_{4} \mathbf{H}_{4} \mathrm{O}_{6}\right)\left(\mathrm{H}_{2} \mathrm{O}\right)_{2} \cdot \mathbf{H}_{2} \mathrm{O}\right]$, 6.

\begin{tabular}{|c|c|c|c|}
\hline Moiety & Distance $(\AA)$ & Moiety & Distance $(\AA)$ \\
\hline $\mathrm{Mg}-\mathrm{O}(8)$ & $2.0063(19)$ & $\mathrm{Mg}-\mathrm{O}(1)$ & $2.0835(17)$ \\
\hline $\mathrm{Mg}-\mathrm{O}(7)$ & $2.0536(18)$ & $\mathrm{Mg}-\mathrm{O}(4)^{\# 1}$ & $2.0944(16)$ \\
\hline $\mathrm{Mg}-\mathrm{O}(5)^{\# 1}$ & $2.0624(16)$ & $\mathrm{O}(4)-\mathrm{Mg}^{\# 2}$ & $2.0944(16)$ \\
\hline $\mathrm{Mg}-\mathrm{O}(3)$ & $2.0631(16)$ & $\mathrm{O}(5)-\mathrm{Mg}^{\# 2}$ & $2.0624(16)$ \\
\hline \multicolumn{4}{|c|}{ Organic and Water } \\
\hline Moiety & Distance $(\AA)$ & Moiety & Distance $(\AA)$ \\
\hline $\mathrm{C}(1)-\mathrm{O}(2)$ & $1.232(3)$ & $\mathrm{C}(4)-\mathrm{O}(4)$ & $1.434(2)$ \\
\hline $\mathrm{C}(1)-\mathrm{O}(1)$ & $1.279(3)$ & $C(4)-C(5)$ & $1.542(3)$ \\
\hline $\mathrm{C}(1)-\mathrm{C}(2)$ & $1.534(3)$ & $\mathrm{C}(4)-\mathrm{H}(4)$ & $0.93(2)$ \\
\hline $\mathrm{C}(2)-\mathrm{O}(3)$ & $1.431(2)$ & $\mathrm{C}(5)-\mathrm{O}(6)$ & $1.249(2)$ \\
\hline$C(2)-C(4)$ & $1.532(3)$ & $\mathrm{C}(5)-\mathrm{O}(5)$ & $1.258(2)$ \\
\hline $\mathrm{C}(2)-\mathrm{H}(2)$ & $0.97(2)$ & $\mathrm{O}(8)-\mathrm{H}(81)$ & $0.77(4)$ \\
\hline $\mathrm{O}(3)-\mathrm{H}(31)$ & $0.84(3)$ & $\mathrm{O}(8)-\mathrm{H}(82)$ & $0.83(4)$ \\
\hline $\mathrm{O}(4)-\mathrm{H}(41)$ & $0.83(3)$ & $\mathrm{O}(9)-\mathrm{H}(91)$ & $0.91(5)$ \\
\hline $\mathrm{O}(7)-\mathrm{H}(71)$ & $0.87(4)$ & $\mathrm{O}(9)-\mathrm{H}(92)$ & $0.79(4)$ \\
\hline $\mathrm{O}(7)-\mathrm{H}(72)$ & $0.82(3)$ & & \\
\hline Moiety & Angle $\left(^{\circ}\right)$ & Moiety & Angle $\left(^{\circ}\right)$ \\
\hline $\mathrm{O}(8)-\mathrm{Mg}-\mathrm{O}(7)$ & $93.44(9)$ & $\mathrm{O}(3)-\mathrm{Mg}-\mathrm{O}(1)$ & 75.74(6) \\
\hline $\mathrm{O}(8)-\mathrm{Mg}-\mathrm{O}(5)^{\# 1}$ & $163.77(8)$ & $\mathrm{O}(8)-\mathrm{Mg}-\mathrm{O}(4)^{\# 1}$ & $88.63(8)$ \\
\hline $\mathrm{O}(7)-\mathrm{Mg}-\mathrm{O}(5)^{\# 1}$ & $88.92(7)$ & $\mathrm{O}(7)-\mathrm{Mg}-\mathrm{O}(4)^{\# 1}$ & $105.18(7)$ \\
\hline $\mathrm{O}(8)-\mathrm{Mg}-\mathrm{O}(3)$ & $99.90(8)$ & $\mathrm{O}(5)^{\# 1}-\mathrm{Mg}-\mathrm{O}(4)^{\# 1}$ & $75.27(6)$ \\
\hline $\mathrm{O}(7)-\mathrm{Mg}-\mathrm{O}(3)$ & $87.59(7)$ & $\mathrm{O}(3)-\mathrm{Mg}-\mathrm{O}(4)^{\# 1}$ & $164.29(7)$ \\
\hline $\mathrm{O}(5)^{\# 1}-\mathrm{Mg}-\mathrm{O}(3)$ & $96.25(7)$ & $\mathrm{O}(1)-\mathrm{Mg}-\mathrm{O}(4)^{\# 1}$ & $90.86(6)$ \\
\hline $\mathrm{O}(8)-\mathrm{Mg}-\mathrm{O}(1)$ & $92.28(8)$ & $\mathrm{C}(1)-\mathrm{O}(1)-\mathrm{Mg}$ & $120.28(13)$ \\
\hline $\mathrm{O}(7)-\mathrm{Mg}-\mathrm{O}(1)$ & $163.08(8)$ & $\mathrm{C}(2)-\mathrm{O}(3)-\mathrm{Mg}$ & $118.87(12)$ \\
\hline $\mathrm{O}(5)^{\# 1}-\mathrm{Mg}-\mathrm{O}(1)$ & $90.03(7)$ & $\mathrm{C}(4)-\mathrm{O}(4)-\mathrm{Mg}^{\# 2}$ & $118.10(12)$ \\
\hline $\mathrm{C}(5)-\mathrm{O}(5)-\mathrm{Mg}^{\# 2}$ & $121.20(13)$ & $\mathrm{Mg}-\mathrm{O}(3)-\mathrm{H}(31)$ & $129.0(19)$ \\
\hline $\mathrm{Mg}^{\# 2}-\mathrm{O}(4)-\mathrm{H}(41)$ & $126.1(17)$ & $\mathrm{Mg}-\mathrm{O}(8)-\mathrm{H}(81)$ & $126(3)$ \\
\hline $\mathrm{Mg}-\mathrm{O}(7)-\mathrm{H}(71)$ & $130(3)$ & $\mathrm{Mg}-\mathrm{O}(8)-\mathrm{H}(82)$ & $115(2)$ \\
\hline $\mathrm{Mg}-\mathrm{O}(7)-\mathrm{H}(72)$ & $122(2)$ & & \\
\hline \multicolumn{4}{|c|}{ Organic } \\
\hline Moiety & Angle $\left(^{\circ}\right)$ & Moiety & Angle $\left(^{\circ}\right)$ \\
\hline $\mathrm{O}(2)-\mathrm{C}(1)-\mathrm{O}(1)$ & $125.0(2)$ & $\mathrm{O}(4)-\mathrm{C}(4)-\mathrm{C}(2)$ & 109.11(15) \\
\hline $\mathrm{O}(2)-\mathrm{C}(1)-\mathrm{C}(2)$ & 119.18(18) & $\mathrm{O}(4)-\mathrm{C}(4)-\mathrm{C}(5)$ & $106.62(15)$ \\
\hline $\mathrm{O}(1)-\mathrm{C}(1)-\mathrm{C}(2)$ & $115.72(17)$ & $C(2)-C(4)-C(5)$ & $112.07(16)$ \\
\hline $\mathrm{O}(3)-\mathrm{C}(2)-\mathrm{C}(4)$ & $111.67(15)$ & $\mathrm{O}(4)-\mathrm{C}(4)-\mathrm{H}(4)$ & 109.3(13) \\
\hline $\mathrm{O}(3)-\mathrm{C}(2)-\mathrm{C}(1)$ & $107.80(16)$ & $\mathrm{C}(2)-\mathrm{C}(4)-\mathrm{H}(4)$ & $110.1(13)$ \\
\hline $\mathrm{C}(4)-\mathrm{C}(2)-\mathrm{C}(1)$ & 114.61(16) & $\mathrm{C}(5)-\mathrm{C}(4)-\mathrm{H}(4)$ & 109.6(13) \\
\hline
\end{tabular}




\begin{tabular}{llll}
$\mathrm{O}(3)-\mathrm{C}(2)-\mathrm{H}(2)$ & $111.2(14)$ & $\mathrm{O}(6)-\mathrm{C}(5)-\mathrm{O}(5)$ & $125.36(18)$ \\
$\mathrm{C}(4)-\mathrm{C}(2)-\mathrm{H}(2)$ & $106.2(14)$ & $\mathrm{O}(6)-\mathrm{C}(5)-\mathrm{C}(4)$ & $117.90(18)$ \\
$\mathrm{C}(1)-\mathrm{C}(2)-\mathrm{H}(2)$ & $105.1(13)$ & $\mathrm{O}(5)-\mathrm{C}(5)-\mathrm{C}(4)$ & $116.74(17)$ \\
$\mathrm{C}(2)-\mathrm{O}(3)-\mathrm{H}(31)$ & $112(2)$ & $\mathrm{C}(4)-\mathrm{O}(4)-\mathrm{H}(41)$ & $112.6(17)$ \\
\hline & \multicolumn{3}{c}{ Water } \\
\hline Moiety & Angle $\left(^{\circ}\right)$ & Moiety & Angle $\left(^{\circ}\right)$ \\
\hline $\mathrm{H}(71)-\mathrm{O}(7)-\mathrm{H}(72)$ & $107(3)$ & $\mathrm{H}(81)-\mathrm{O}(8)-\mathrm{H}(82)$ & $113(3)$ \\
$\mathrm{H}(91)-\mathrm{O}(9)-\mathrm{H}(92)$ & $104(4)$ & & \\
\hline
\end{tabular}

Symmetry transformations used to generate equivalent atoms:

$\# 1-x+1 / 2, y-1 / 2,-z+3 / 2 \quad \# 2-x+1 / 2, y+1 / 2,-z+3 / 2$ 
Table S13: Atomic coordinates (x 10 ${ }^{4}$ ) and equivalent isotropic displacement parameters $\left(\AA^{2} \times 10^{3}\right)$ for $\left[\mathrm{Mg}\left(\right.\right.$ meso- $\left.\left.\mathrm{C}_{4} \mathrm{H}_{4} \mathrm{O}_{6}\right)\left(\mathrm{H}_{2} \mathrm{O}\right)_{2} \cdot \mathrm{H}_{2} \mathrm{O}\right], 7$.

\begin{tabular}{lllll}
\hline Atom & $\mathrm{x}$ & $\mathrm{y}$ & $\mathrm{z}$ & $\mathrm{U}(\mathrm{eq})$ \\
\hline $\mathrm{C}(1)$ & $571(3)$ & $5099(3)$ & $2576(2)$ & $23(1)$ \\
$\mathrm{C}(2)$ & $1936(3)$ & $4563(2)$ & $2400(2)$ & $21(1)$ \\
$\mathrm{C}(3)$ & $1867(3)$ & $3192(2)$ & $2298(2)$ & $21(1)$ \\
$\mathrm{C}(4)$ & $1080(3)$ & $2852(2)$ & $1467(2)$ & $23(1)$ \\
$\mathrm{Mg}$ & $1438(1)$ & $6576(1)$ & $1133(1)$ & $24(1)$ \\
$\mathrm{O}(1)$ & $102(2)$ & $5807(2)$ & $2016(1)$ & $27(1)$ \\
$\mathrm{O}(2)$ & $18(2)$ & $4811(2)$ & $3304(1)$ & $28(1)$ \\
$\mathrm{O}(3)$ & $2456(2)$ & $5086(2)$ & $1605(1)$ & $26(1)$ \\
$\mathrm{O}(4)$ & $3159(2)$ & $2705(2)$ & $2202(1)$ & $26(1)$ \\
$\mathrm{O}(5)$ & $1697(2)$ & $2350(2)$ & $836(1)$ & $27(1)$ \\
$\mathrm{O}(6)$ & $-128(2)$ & $3120(2)$ & $1457(1)$ & $27(1)$ \\
$\mathrm{O}(7)$ & $134(2)$ & $7832(2)$ & $640(2)$ & $34(1)$ \\
$\mathrm{O}(8)$ & $1343(3)$ & $5814(3)$ & $-77(2)$ & $44(1)$ \\
$\mathrm{O}(9)$ & $2729(4)$ & $3898(3)$ & $9334(2)$ & $80(1)$ \\
\hline
\end{tabular}


Table S14. Bond lengths $[\AA]$ and Bond angles $\left[{ }^{\circ}\right]$ for $\left[\mathrm{Mg}\left(\right.\right.$ meso $\left.\left.-\mathrm{C}_{4} \mathbf{H}_{4} \mathrm{O}_{6}\right)\left(\mathrm{H}_{2} \mathrm{O}\right)_{2} \cdot \mathbf{H}_{2} \mathrm{O}\right]$, 7.

\begin{tabular}{|c|c|c|c|}
\hline Moiety & Distance $(\AA)$ & Moiety & Distance $(\AA)$ \\
\hline $\mathrm{Mg}-\mathrm{O}(8)$ & $2.000(3)$ & $\mathrm{Mg}-\mathrm{O}(3)$ & $2.086(2)$ \\
\hline $\mathrm{Mg}-\mathrm{O}(7)$ & $2.062(3)$ & $\mathrm{Mg}-\mathrm{O}(5)^{\# 1}$ & $2.116(2)$ \\
\hline $\mathrm{Mg}-\mathrm{O}(1)$ & $2.071(2)$ & $\mathrm{O}(4)-\mathrm{Mg}^{\# 2}$ & $2.079(2)$ \\
\hline $\mathrm{Mg}-\mathrm{O}(4)^{\# 1}$ & $2.079(2)$ & $\mathrm{O}(5)-\mathrm{Mg}^{\# 2}$ & $2.116(2)$ \\
\hline $\mathrm{C}(1)-\mathrm{Mg}$ & $2.856(3)$ & & \\
\hline \multicolumn{4}{|c|}{ Organic and Water } \\
\hline Moiety & Distance $(\AA)$ & Moiety & Distance $(\AA)$ \\
\hline $\mathrm{C}(1)-\mathrm{O}(1)$ & $1.246(3)$ & $\mathrm{C}(3)-\mathrm{O}(4)$ & $1.418(4)$ \\
\hline $\mathrm{C}(1)-\mathrm{O}(2)$ & $1.262(3)$ & $C(3)-C(4)$ & $1.519(4)$ \\
\hline $\mathrm{C}(1)-\mathrm{C}(2)$ & $1.522(4)$ & $\mathrm{C}(3)-\mathrm{H}(3)$ & $0.94(3)$ \\
\hline $\mathrm{C}(2)-\mathrm{O}(3)$ & $1.423(3)$ & $\mathrm{C}(4)-\mathrm{O}(6)$ & $1.252(3)$ \\
\hline $\mathrm{C}(2)-\mathrm{C}(3)$ & $1.549(4)$ & $\mathrm{C}(4)-\mathrm{O}(5)$ & $1.261(3)$ \\
\hline $\mathrm{C}(2)-\mathrm{H}(2)$ & $0.96(3)$ & $\mathrm{O}(3)-\mathrm{H}(31)$ & $0.85(4)$ \\
\hline $\mathrm{O}(4)-\mathrm{H}(41)$ & $0.82(4)$ & $\mathrm{O}(8)-\mathrm{H}(81)$ & $0.94(5)$ \\
\hline $\mathrm{O}(7)-\mathrm{H}(71)$ & $0.91(4)$ & $\mathrm{O}(8)-\mathrm{H}(82)$ & $0.70(6)$ \\
\hline $\mathrm{O}(7)-\mathrm{H}(72)$ & $0.82(5)$ & $\mathrm{O}(9)-\mathrm{H}(91)$ & $0.856(11)$ \\
\hline $\mathrm{O}(9)-\mathrm{H}(92)$ & $0.852(10)$ & & \\
\hline Moiety & Angle $\left(^{\circ}\right)$ & Moiety & Angle $\left(^{\circ}\right)$ \\
\hline $\mathrm{O}(1)-\mathrm{C}(1)-\mathrm{Mg}$ & $40.44(14)$ & $\mathrm{O}(8)-\mathrm{Mg}-\mathrm{O}(3)$ & $89.13(11)$ \\
\hline $\mathrm{O}(2)-\mathrm{C}(1)-\mathrm{Mg}$ & $158.8(2)$ & $\mathrm{O}(7)-\mathrm{Mg}-\mathrm{O}(3)$ & $169.21(11)$ \\
\hline $\mathrm{C}(2)-\mathrm{C}(1)-\mathrm{Mg}$ & $79.90(16)$ & $\mathrm{O}(1)-\mathrm{Mg}-\mathrm{O}(3)$ & $76.61(9)$ \\
\hline $\mathrm{O}(8)-\mathrm{Mg}-\mathrm{O}(7)$ & $86.62(12)$ & $\mathrm{O}(4)^{\# 1}-\mathrm{Mg}-\mathrm{O}(3)$ & $97.72(9)$ \\
\hline $\mathrm{O}(8)-\mathrm{Mg}-\mathrm{O}(1)$ & $111.39(11)$ & $\mathrm{O}(8)-\mathrm{Mg}-\mathrm{O}(5)^{\# 1}$ & $91.71(11)$ \\
\hline $\mathrm{O}(7)-\mathrm{Mg}-\mathrm{O}(1)$ & $95.73(10)$ & $\mathrm{O}(7)-\mathrm{Mg}-\mathrm{O}(5)^{\# 1}$ & $102.03(10)$ \\
\hline $\mathrm{O}(8)-\mathrm{Mg}-\mathrm{O}(4)^{\# 1}$ & $164.48(12)$ & $\mathrm{O}(1)-\mathrm{Mg}-\mathrm{O}(5)^{\# 1}$ & $151.66(9)$ \\
\hline $\mathrm{O}(7)-\mathrm{Mg}-\mathrm{O}(4)^{\# 1}$ & $88.86(10)$ & $\mathrm{O}(4)^{\# 1}-\mathrm{Mg}-\mathrm{O}(5)^{\# 1}$ & $74.73(9)$ \\
\hline $\mathrm{O}(1)-\mathrm{Mg}-\mathrm{O}(4)^{\# 1}$ & $83.82(9)$ & $\mathrm{O}(3)-\mathrm{Mg}-\mathrm{O}(5)^{\# 1}$ & $87.99(9)$ \\
\hline $\mathrm{O}(8)-\mathrm{Mg}-\mathrm{C}(1)$ & $114.64(11)$ & $\mathrm{C}(1)-\mathrm{O}(1)-\mathrm{Mg}$ & $116.58(19)$ \\
\hline $\mathrm{O}(7)-\mathrm{Mg}-\mathrm{C}(1)$ & $118.20(10)$ & $\mathrm{C}(2)-\mathrm{O}(3)-\mathrm{Mg}$ & $115.67(17)$ \\
\hline $\mathrm{O}(1)-\mathrm{Mg}-\mathrm{C}(1)$ & $22.97(8)$ & $\mathrm{Mg}-\mathrm{O}(3)-\mathrm{H}(31)$ & $119(3)$ \\
\hline $\mathrm{O}(4)^{\# 1}-\mathrm{Mg}-\mathrm{C}(1)$ & $80.52(9)$ & $\mathrm{C}(3)-\mathrm{O}(4)-\mathrm{Mg}^{\# 2}$ & $119.45(18)$ \\
\hline $\mathrm{O}(3)-\mathrm{Mg}-\mathrm{C}(1)$ & $55.12(8)$ & $\mathrm{Mg}^{\mathrm{f} 2}-\mathrm{O}(4)-\mathrm{H}(41)$ & $124(3)$ \\
\hline $\mathrm{O}(5)^{\# 1}-\mathrm{Mg}-\mathrm{C}(1)$ & $131.88(9)$ & $\mathrm{C}(4)-\mathrm{O}(5)-\mathrm{Mg}^{\# 2}$ & $117.65(18)$ \\
\hline $\mathrm{Mg}-\mathrm{O}(7)-\mathrm{H}(71)$ & $123(2)$ & $\mathrm{Mg}-\mathrm{O}(8)-\mathrm{H}(81)$ & $127(3)$ \\
\hline $\mathrm{Mg}-\mathrm{O}(7)-\mathrm{H}(72)$ & $128(3)$ & $\mathrm{Mg}-\mathrm{O}(8)-\mathrm{H}(82)$ & $132(5)$ \\
\hline \multicolumn{4}{|c|}{ Organic } \\
\hline Moiety & Angle $\left(^{\circ}\right)$ & Moiety & Angle $\left(^{\circ}\right)$ \\
\hline $\mathrm{O}(1)-\mathrm{C}(1)-\mathrm{O}(2)$ & $125.0(3)$ & $\mathrm{O}(4)-\mathrm{C}(3)-\mathrm{C}(4)$ & $107.4(2)$ \\
\hline
\end{tabular}




\begin{tabular}{llll}
$\mathrm{O}(1)-\mathrm{C}(1)-\mathrm{C}(2)$ & $118.6(3)$ & $\mathrm{O}(4)-\mathrm{C}(3)-\mathrm{C}(2)$ & $110.6(2)$ \\
$\mathrm{O}(2)-\mathrm{C}(1)-\mathrm{C}(2)$ & $116.4(3)$ & $\mathrm{C}(4)-\mathrm{C}(3)-\mathrm{C}(2)$ & $110.7(2)$ \\
$\mathrm{O}(3)-\mathrm{C}(2)-\mathrm{C}(1)$ & $108.1(2)$ & $\mathrm{O}(4)-\mathrm{C}(3)-\mathrm{H}(3)$ & $111.6(16)$ \\
$\mathrm{O}(3)-\mathrm{C}(2)-\mathrm{C}(3)$ & $110.2(2)$ & $\mathrm{C}(4)-\mathrm{C}(3)-\mathrm{H}(3)$ & $109.2(16)$ \\
$\mathrm{C}(1)-\mathrm{C}(2)-\mathrm{C}(3)$ & $111.7(2)$ & $\mathrm{C}(2)-\mathrm{C}(3)-\mathrm{H}(3)$ & $107.4(17)$ \\
$\mathrm{O}(3)-\mathrm{C}(2)-\mathrm{H}(2)$ & $112.2(18)$ & $\mathrm{O}(6)-\mathrm{C}(4)-\mathrm{O}(5)$ & $125.2(3)$ \\
$\mathrm{C}(1)-\mathrm{C}(2)-\mathrm{H}(2)$ & $106.0(17)$ & $\mathrm{O}(6)-\mathrm{C}(4)-\mathrm{C}(3)$ & $117.0(3)$ \\
$\mathrm{C}(3)-\mathrm{C}(2)-\mathrm{H}(2)$ & $108.6(17)$ & $\mathrm{O}(5)-\mathrm{C}(4)-\mathrm{C}(3)$ & $117.7(3)$ \\
$\mathrm{C}(2)-\mathrm{O}(3)-\mathrm{H}(31)$ & $107(3)$ & $\mathrm{C}(3)-\mathrm{O}(4)-\mathrm{H}(41)$ & $116(3)$ \\
\hline & & Water & Angle $\left(^{\circ}\right)$ \\
\hline $\mathrm{Moiety}$ & Angle $\left(^{\circ}\right)$ & Moiety & $99.0(16)$ \\
$\mathrm{H}(71)-\mathrm{O}(7)-\mathrm{H}(72)$ & $108(4)$ & $\mathrm{H}(91)-\mathrm{O}(9)-\mathrm{H}(92)$ & \\
$\mathrm{H}(81)-\mathrm{O}(8)-\mathrm{H}(82)$ & $100(5)$ & &
\end{tabular}

Symmetry transformations used to generate equivalent atoms:

$\# 1-\mathrm{x}+1 / 2, \mathrm{y}+1 / 2, \mathrm{z} \quad \# 2-\mathrm{x}+1 / 2, \mathrm{y}-1 / 2, \mathrm{z}$ 
Table S15: Atomic coordinates (x 104) and equivalent isotropic displacement parameters $\left(\AA^{2} \times 10^{3}\right)$ for $\left[\mathrm{Mg}\left(\right.\right.$ meso- $\left.\left.\mathrm{C}_{4} \mathrm{H}_{4} \mathrm{O}_{6}\right)\left(\mathrm{H}_{2} \mathrm{O}\right)_{2}\right], 8$.

\begin{tabular}{lllll}
\hline Atom & $\mathrm{x}$ & $\mathrm{y}$ & $\mathrm{z}$ & $\mathrm{U}(\mathrm{eq})$ \\
\hline $\mathrm{C}(1)$ & $3495(3)$ & $6598(1)$ & $9833(2)$ & $20(1)$ \\
$\mathrm{C}(2)$ & $3617(3)$ & $7547(1)$ & $10733(2)$ & $19(1)$ \\
$\mathrm{C}(3)$ & $3324(3)$ & $8572(1)$ & $10000(2)$ & $18(1)$ \\
$\mathrm{C}(4)$ & $5448(3)$ & $8804(1)$ & $9337(2)$ & $20(1)$ \\
$\mathrm{Mg}$ & $1696(1)$ & $5851(1)$ & $12111(1)$ & $19(1)$ \\
$\mathrm{O}(1)$ & $2725(2)$ & $5792(1)$ & $10294(1)$ & $23(1)$ \\
$\mathrm{O}(2)$ & $4252(2)$ & $6669(1)$ & $8768(1)$ & $27(1)$ \\
$\mathrm{O}(3)$ & $1933(2)$ & $7429(1)$ & $11593(1)$ & $22(1)$ \\
$\mathrm{O}(4)$ & $1288(2)$ & $8630(1)$ & $9020(1)$ & $22(1)$ \\
$\mathrm{O}(5)$ & $7391(2)$ & $8783(1)$ & $10055(1)$ & $28(1)$ \\
$\mathrm{O}(6)$ & $5064(2)$ & $9034(1)$ & $8132(1)$ & $24(1)$ \\
$\mathrm{O}(7)$ & $1986(3)$ & $4265(1)$ & $12256(2)$ & $29(1)$ \\
$\mathrm{O}(8)$ & $-1731(2)$ & $5747(1)$ & $11532(2)$ & $27(1)$ \\
\hline
\end{tabular}


Table S16. Bond lengths $[\AA]$ and Bond angles $\left[{ }^{\circ}\right]$ for $\left[\mathrm{Mg}\left(\boldsymbol{m e s o}-\mathrm{C}_{4} \mathrm{H}_{4} \mathrm{O}_{6}\right)\left(\mathrm{H}_{2} \mathrm{O}\right)_{2}\right], 8$.

\begin{tabular}{|c|c|c|c|}
\hline Moiety & Distance $(\AA)$ & Moiety & Distance $(\AA)$ \\
\hline $\mathrm{Mg}-\mathrm{O}(8)$ & $1.9951(15)$ & $\mathrm{Mg}-\mathrm{O}(4)^{\# 1}$ & $2.1147(15)$ \\
\hline $\mathrm{Mg}-\mathrm{O}(1)$ & $2.0443(15)$ & $\mathrm{Mg}-\mathrm{O}(3)$ & $2.1344(14)$ \\
\hline $\mathrm{Mg}-\mathrm{O}(6)^{\# 1}$ & $2.0752(15)$ & $\mathrm{O}(4)-\mathrm{Mg}^{\# 2}$ & $2.1147(15)$ \\
\hline $\mathrm{Mg}-\mathrm{O}(7)$ & $2.0778(16)$ & $\mathrm{O}(6)-\mathrm{Mg}^{\# 2}$ & $2.0752(15)$ \\
\hline \multicolumn{4}{|c|}{ Organic and Water } \\
\hline Moiety & Distance $(\AA)$ & Moiety & Distance $(\AA)$ \\
\hline $\mathrm{C}(1)-\mathrm{O}(2)$ & $1.242(2)$ & $\mathrm{C}(3)-\mathrm{O}(4)$ & $1.427(2)$ \\
\hline $\mathrm{C}(1)-\mathrm{O}(1)$ & $1.264(2)$ & $C(3)-C(4)$ & $1.535(2)$ \\
\hline $\mathrm{C}(1)-\mathrm{C}(2)$ & $1.537(2)$ & $\mathrm{C}(3)-\mathrm{H}(3)$ & $0.942(17)$ \\
\hline $\mathrm{C}(2)-\mathrm{O}(3)$ & $1.428(2)$ & $\mathrm{C}(4)-\mathrm{O}(5)$ & $1.247(2)$ \\
\hline$C(2)-C(3)$ & $1.528(2)$ & $\mathrm{C}(4)-\mathrm{O}(6)$ & $1.254(2)$ \\
\hline $\mathrm{C}(2)-\mathrm{H}(2)$ & $1.01(2)$ & $\mathrm{O}(3)-\mathrm{H}(31)$ & $0.83(3)$ \\
\hline $\mathrm{O}(4)-\mathrm{H}(41)$ & $0.89(3)$ & $\mathrm{O}(8)-\mathrm{H}(81)$ & $0.82(3)$ \\
\hline $\mathrm{O}(7)-\mathrm{H}(71)$ & $0.80(3)$ & $\mathrm{O}(8)-\mathrm{H}(82)$ & $0.84(3)$ \\
\hline $\mathrm{O}(7)-\mathrm{H}(72)$ & $0.81(3)$ & & \\
\hline Moiety & Angle $\left(^{\circ}\right)$ & Moiety & Angle $\left(^{\circ}\right)$ \\
\hline $\mathrm{O}(8)-\mathrm{Mg}-\mathrm{O}(1)$ & $98.89(6)$ & $\mathrm{O}(8)-\mathrm{Mg}-\mathrm{O}(3)$ & $95.37(6)$ \\
\hline $\mathrm{O}(8)-\mathrm{Mg}-\mathrm{O}(6)^{\# 1}$ & $167.26(6)$ & $\mathrm{O}(1)-\mathrm{Mg}-\mathrm{O}(3)$ & $76.72(5)$ \\
\hline $\mathrm{O}(1)-\mathrm{Mg}-\mathrm{O}(6)^{\# 1}$ & $93.85(6)$ & $\mathrm{O}(6)^{\# 1}-\mathrm{Mg}-\mathrm{O}(3)$ & $87.79(5)$ \\
\hline $\mathrm{O}(8)-\mathrm{Mg}-\mathrm{O}(7)$ & $91.14(6)$ & $\mathrm{O}(7)-\mathrm{Mg}-\mathrm{O}(3)$ & 165.69(6) \\
\hline $\mathrm{O}(1)-\mathrm{Mg}-\mathrm{O}(7)$ & $89.71(6)$ & $\mathrm{O}(4)^{\# 1}-\mathrm{Mg}-\mathrm{O}(3)$ & $86.70(5)$ \\
\hline $\mathrm{O}(6)^{\# 1}-\mathrm{Mg}-\mathrm{O}(7)$ & $88.63(6)$ & $\mathrm{C}(1)-\mathrm{O}(1)-\mathrm{Mg}$ & $118.79(11)$ \\
\hline $\mathrm{O}(8)-\mathrm{Mg}-\mathrm{O}(4)^{\# 1}$ & $92.24(6)$ & $\mathrm{C}(2)-\mathrm{O}(3)-\mathrm{Mg}$ & $109.59(9)$ \\
\hline $\mathrm{O}(1)-\mathrm{Mg}-\mathrm{O}(4)^{\# 1}$ & $160.76(5)$ & $\mathrm{C}(2)-\mathrm{O}(3)-\mathrm{H}(31)$ & 103.4(19) \\
\hline $\mathrm{O}(6)^{\# 1}-\mathrm{Mg}-\mathrm{O}(4)^{\# 1}$ & $75.59(5)$ & $\mathrm{Mg}-\mathrm{O}(3)-\mathrm{H}(31)$ & $105.9(19)$ \\
\hline $\mathrm{O}(7)-\mathrm{Mg}-\mathrm{O}(4)^{\# 1}$ & $105.79(6)$ & $\mathrm{C}(3)-\mathrm{O}(4)-\mathrm{Mg}^{\mathrm{H2}}$ & $117.44(10)$ \\
\hline $\mathrm{Mg}^{\# 2}-\mathrm{O}(4)-\mathrm{H}(41)$ & $129.5(15)$ & $\mathrm{C}(4)-\mathrm{O}(6)-\mathrm{Mg}^{\# 2}$ & $121.09(10)$ \\
\hline $\mathrm{Mg}-\mathrm{O}(7)-\mathrm{H}(71)$ & $117.9(18)$ & $\mathrm{Mg}-\mathrm{O}(8)-\mathrm{H}(81)$ & $116.2(18)$ \\
\hline $\mathrm{Mg}-\mathrm{O}(7)-\mathrm{H}(72)$ & $118(2)$ & $\mathrm{Mg}-\mathrm{O}(8)-\mathrm{H}(82)$ & $114.9(18)$ \\
\hline \multicolumn{4}{|c|}{ Organic } \\
\hline Moiety & Angle $\left(^{\circ}\right)$ & Moiety & Angle $\left(^{\circ}\right)$ \\
\hline $\mathrm{O}(2)-\mathrm{C}(1)-\mathrm{O}(1)$ & 125.91(16) & $\mathrm{O}(4)-\mathrm{C}(3)-\mathrm{C}(2)$ & $113.96(14)$ \\
\hline $\mathrm{O}(2)-\mathrm{C}(1)-\mathrm{C}(2)$ & $118.45(15)$ & $\mathrm{O}(4)-\mathrm{C}(3)-\mathrm{C}(4)$ & $108.57(13)$ \\
\hline $\mathrm{O}(1)-\mathrm{C}(1)-\mathrm{C}(2)$ & $115.53(14)$ & $\mathrm{C}(2)-\mathrm{C}(3)-\mathrm{C}(4)$ & $110.77(13)$ \\
\hline $\mathrm{O}(3)-\mathrm{C}(2)-\mathrm{C}(3)$ & $111.28(13)$ & $\mathrm{O}(4)-\mathrm{C}(3)-\mathrm{H}(3)$ & 107.1(11) \\
\hline $\mathrm{O}(3)-\mathrm{C}(2)-\mathrm{C}(1)$ & 108.18(13) & $\mathrm{C}(2)-\mathrm{C}(3)-\mathrm{H}(3)$ & $107.9(10)$ \\
\hline $\mathrm{C}(3)-\mathrm{C}(2)-\mathrm{C}(1)$ & $114.75(14)$ & $\mathrm{C}(4)-\mathrm{C}(3)-\mathrm{H}(3)$ & $108.4(10)$ \\
\hline $\mathrm{O}(3)-\mathrm{C}(2)-\mathrm{H}(2)$ & 108.3(11) & $\mathrm{O}(5)-\mathrm{C}(4)-\mathrm{O}(6)$ & $125.82(15)$ \\
\hline $\mathrm{C}(3)-\mathrm{C}(2)-\mathrm{H}(2)$ & 109.7(11) & $\mathrm{O}(5)-\mathrm{C}(4)-\mathrm{C}(3)$ & $117.22(15)$ \\
\hline
\end{tabular}




\begin{tabular}{llll}
$\mathrm{C}(1)-\mathrm{C}(2)-\mathrm{H}(2)$ & $104.3(11)$ & $\mathrm{O}(6)-\mathrm{C}(4)-\mathrm{C}(3)$ & $116.90(14)$ \\
$\mathrm{C}(3)-\mathrm{O}(4)-\mathrm{H}(41)$ & $111.3(15)$ & & \\
\hline & & Water & Angle $\left(^{\circ}\right)$ \\
\hline Moiety & Angle $\left(^{\circ}\right)$ & Moiety & $109(2)$ \\
\hline $\mathrm{H}(71)-\mathrm{O}(7)-\mathrm{H}(72)$ & $113(3)$ & $\mathrm{H}(81)-\mathrm{O}(8)-\mathrm{H}(82)$ & \\
\hline
\end{tabular}

Symmetry transformations used to generate equivalent atoms:

$\# 1 \mathrm{x},-\mathrm{y}+3 / 2, \mathrm{z}+1 / 2 \quad \# 2 \mathrm{x},-\mathrm{y}+3 / 2, \mathrm{z}-1 / 2$ 
Table S17: Atomic coordinates (x 104) and equivalent isotropic displacement parameters $\left(\AA^{2} \times 10^{3}\right)$ for $\left[\mathbf{M g}\left(\right.\right.$ meso- $\left.\left.\mathbf{C}_{4} \mathbf{H}_{4} \mathbf{O}_{6}\right)\right], 9$.

\begin{tabular}{lllll}
\hline Atom & $\mathrm{x}$ & $\mathrm{y}$ & $\mathrm{z}$ & $\mathrm{U}(\mathrm{eq})$ \\
\hline $\mathrm{C}(1)$ & $2298(6)$ & $3735(5)$ & $1594(5)$ & $27(1)$ \\
$\mathrm{C}(2)$ & $1575(6)$ & $2870(5)$ & $160(5)$ & $28(1)$ \\
$\mathrm{Mg}$ & 0 & $1491(2)$ & 2500 & $30(1)$ \\
$\mathrm{O}(1)$ & $2215(4)$ & $3112(3)$ & $2755(3)$ & $33(1)$ \\
$\mathrm{O}(2)$ & $2927(5)$ & $4985(3)$ & $1522(3)$ & $40(1)$ \\
$\mathrm{O}(3)$ & $215(5)$ & $1768(3)$ & $377(3)$ & $33(1)$ \\
\hline
\end{tabular}


Table S18. Bond lengths $[\AA]$ and Bond angles $\left[{ }^{\circ}\right]$ for $\left[\mathrm{Mg}\left(\boldsymbol{m e s o}-\mathrm{C}_{4} \mathrm{H}_{4} \mathrm{O}_{6}\right)\right]$, 9 .

\begin{tabular}{|c|c|c|c|}
\hline Moiety & Distance $(\AA)$ & Moiety & Distance $(\AA)$ \\
\hline $\mathrm{Mg}-\mathrm{O}(2)^{\# 2}$ & $1.995(3)$ & $\mathrm{Mg}-\mathrm{O}(3)^{\# 4}$ & $2.075(3)$ \\
\hline $\mathrm{Mg}-\mathrm{O}(2)^{\# 3}$ & $1.995(3)$ & $\mathrm{Mg}-\mathrm{O}(1)$ & $2.090(3)$ \\
\hline $\mathrm{Mg}-\mathrm{O}(3)$ & $2.075(3)$ & $\mathrm{Mg}-\mathrm{O}(1)^{\# 4}$ & $2.090(3)$ \\
\hline $\mathrm{O}(2)-\mathrm{Mg}^{\# 5}$ & $1.995(3)$ & & \\
\hline \multicolumn{4}{|c|}{ Organic } \\
\hline Moiety & Distance $(\AA)$ & Moiety & Distance $(\AA)$ \\
\hline $\mathrm{C}(1)-\mathrm{O}(2)$ & $1.238(5)$ & $\mathrm{C}(2)-\mathrm{O}(3)$ & $1.436(5)$ \\
\hline $\mathrm{C}(1)-\mathrm{O}(1)$ & $1.256(5)$ & $C(2)-C(2)^{\# 1}$ & $1.552(8)$ \\
\hline $\mathrm{C}(1)-\mathrm{C}(2)$ & $1.517(6)$ & $\mathrm{C}(2)-\mathrm{H}(2)$ & $1.109(10)$ \\
\hline $\mathrm{O}(3)-\mathrm{H}(3)$ & $0.847(10)$ & & \\
\hline Moiety & Angle $\left(^{\circ}\right)$ & Moiety & Angle $\left(^{\circ}\right)$ \\
\hline $\mathrm{O}(2)^{\# 2}-\mathrm{Mg}-\mathrm{O}(2)^{\# 3}$ & $92.0(2)$ & $\mathrm{O}(2)^{\# 2}-\mathrm{Mg}-\mathrm{O}(1)^{\# 4}$ & $93.10(13)$ \\
\hline $\mathrm{O}(2)^{\# 2}-\mathrm{Mg}-\mathrm{O}(3)$ & $83.82(13)$ & $\mathrm{O}(2)^{\# 3}-\mathrm{Mg}-\mathrm{O}(1)^{\# 4}$ & $159.66(12)$ \\
\hline $\mathrm{O}(2)^{\# 3}-\mathrm{Mg}-\mathrm{O}(3)$ & $106.18(14)$ & $\mathrm{O}(3)-\mathrm{Mg}-\mathrm{O}(1)^{\# 4}$ & $93.95(13)$ \\
\hline $\mathrm{O}(2)^{\# 2}-\mathrm{Mg}-\mathrm{O}(3)^{\# 4}$ & $106.18(14)$ & $\mathrm{O}(3)^{\# 4}-\mathrm{Mg}-\mathrm{O}(1)^{\# 4}$ & $75.84(12)$ \\
\hline $\mathrm{O}(2)^{\# 3}-\mathrm{Mg}-\mathrm{O}(3)^{\# 4}$ & $83.82(13)$ & $\mathrm{O}(1)-\mathrm{Mg}-\mathrm{O}(1)^{\# 4}$ & $88.90(18)$ \\
\hline $\mathrm{O}(3)-\mathrm{Mg}-\mathrm{O}(3)^{\# 4}$ & $165.9(2)$ & $\mathrm{C}(1)-\mathrm{O}(1)-\mathrm{Mg}$ & $117.0(3)$ \\
\hline $\mathrm{O}(2)^{\# 2}-\mathrm{Mg}-\mathrm{O}(1)$ & $159.66(12)$ & $\mathrm{C}(1)-\mathrm{O}(2)-\mathrm{Mg}^{\# 5}$ & $146.2(3)$ \\
\hline $\mathrm{O}(2)^{\# 3}-\mathrm{Mg}-\mathrm{O}(1)$ & $93.10(13)$ & $\mathrm{C}(2)-\mathrm{O}(3)-\mathrm{Mg}$ & 118.1(2) \\
\hline $\mathrm{O}(3)-\mathrm{Mg}-\mathrm{O}(1)$ & $75.84(12)$ & $\mathrm{Mg}-\mathrm{O}(3)-\mathrm{H}(3)$ & $126(4)$ \\
\hline $\mathrm{O}(3)^{\# 4}-\mathrm{Mg}-\mathrm{O}(1)$ & $93.95(13)$ & & \\
\hline \multicolumn{4}{|c|}{ Organic } \\
\hline Moiety & Angle $\left(^{\circ}\right)$ & Moiety & Angle $\left(^{\circ}\right)$ \\
\hline$\overline{\mathrm{O}(2)-\mathrm{C}(1)-\mathrm{O}(1)}$ & $125.7(4)$ & $\mathrm{C}(1)-\mathrm{C}(2)-\mathrm{C}(2)^{\# 1}$ & $110.2(4)$ \\
\hline $\mathrm{O}(2)-\mathrm{C}(1)-\mathrm{C}(2)$ & $117.5(4)$ & $\mathrm{O}(3)-\mathrm{C}(2)-\mathrm{H}(2)$ & $113.4(16)$ \\
\hline $\mathrm{O}(1)-\mathrm{C}(1)-\mathrm{C}(2)$ & $116.8(4)$ & $\mathrm{C}(1)-\mathrm{C}(2)-\mathrm{H}(2)$ & 111.1(17) \\
\hline $\mathrm{O}(3)-\mathrm{C}(2)-\mathrm{C}(1)$ & 106.8(3) & $\mathrm{C}(2)^{\# 1}-\mathrm{C}(2)-\mathrm{H}(2)$ & 106.3(18) \\
\hline $\mathrm{O}(3)-\mathrm{C}(2)-\mathrm{C}(2)^{\# 1}$ & $109.0(4)$ & $\mathrm{C}(2)-\mathrm{O}(3)-\mathrm{H}(3)$ & $101(5)$ \\
\hline
\end{tabular}

Symmetry transformations used to generate equivalent atoms:

$\# 1-x+1 / 2,-y+1 / 2,-\mathrm{z} \quad \# 2 \mathrm{x}-1 / 2, \mathrm{y}-1 / 2, \mathrm{z} \quad \# 3-\mathrm{x}+1 / 2, \mathrm{y}-1 / 2,-\mathrm{z}+1 / 2 \quad \# 4-\mathrm{x}, \mathrm{y},-\mathrm{z}+1 / 2$ $\# 5 \mathrm{x}+1 / 2, \mathrm{y}+1 / 2, \mathrm{z}$ 
Figure S1. X-ray powder diffraction for Structure 1 and 2.

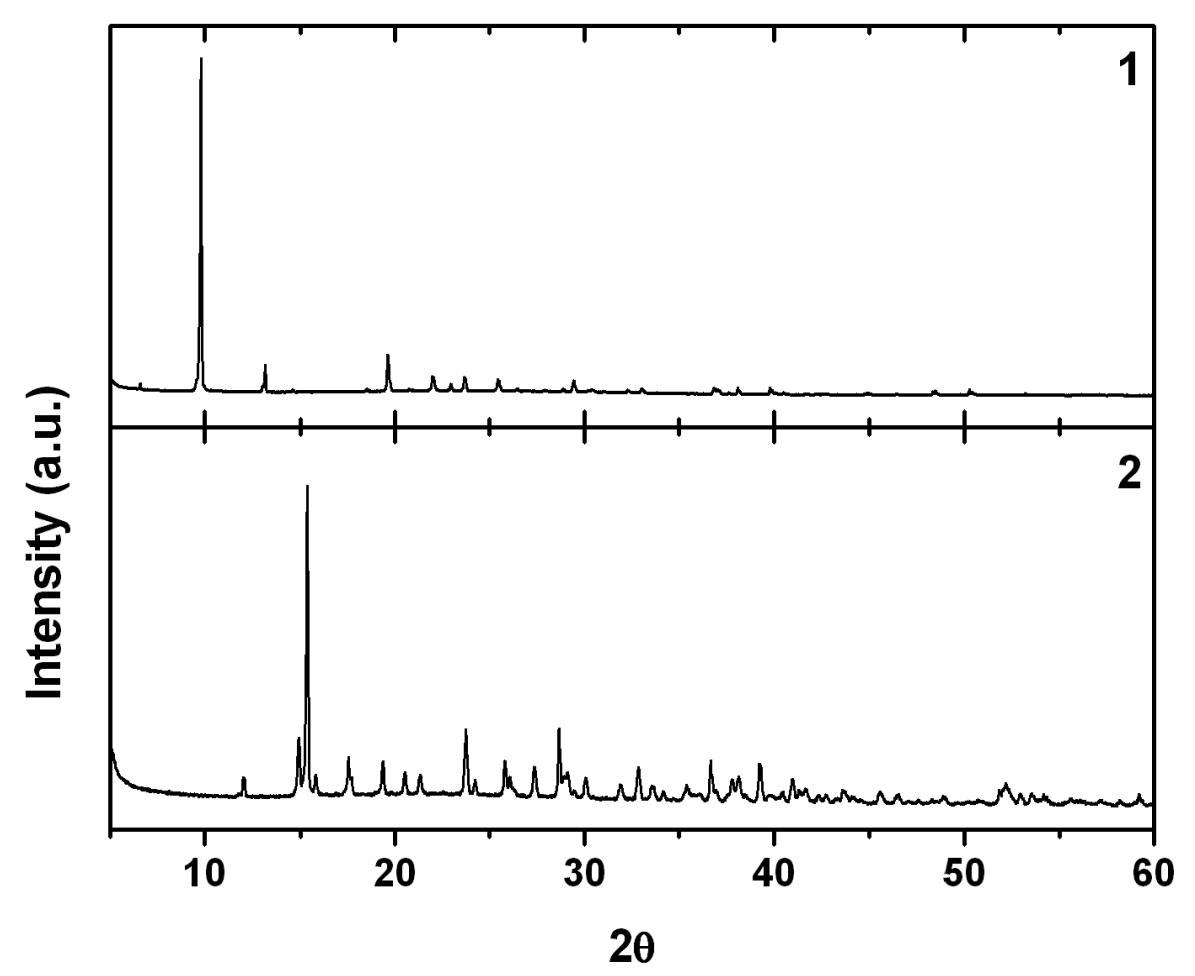


Figure S2. X-ray powder diffraction for Structure 3, 4, and 5.

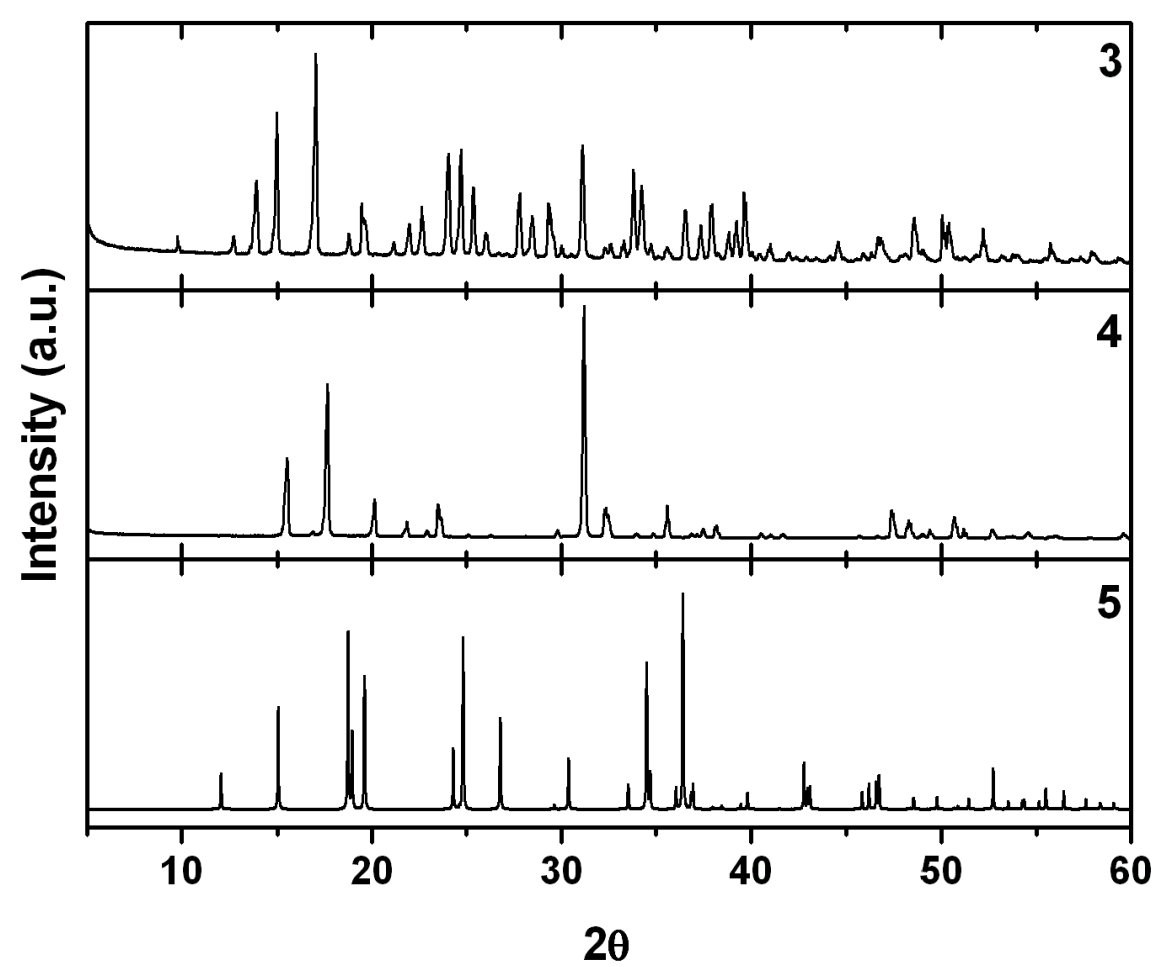


Figure S3. X-ray powder diffraction for Structure 6, 7, 8, and 9.

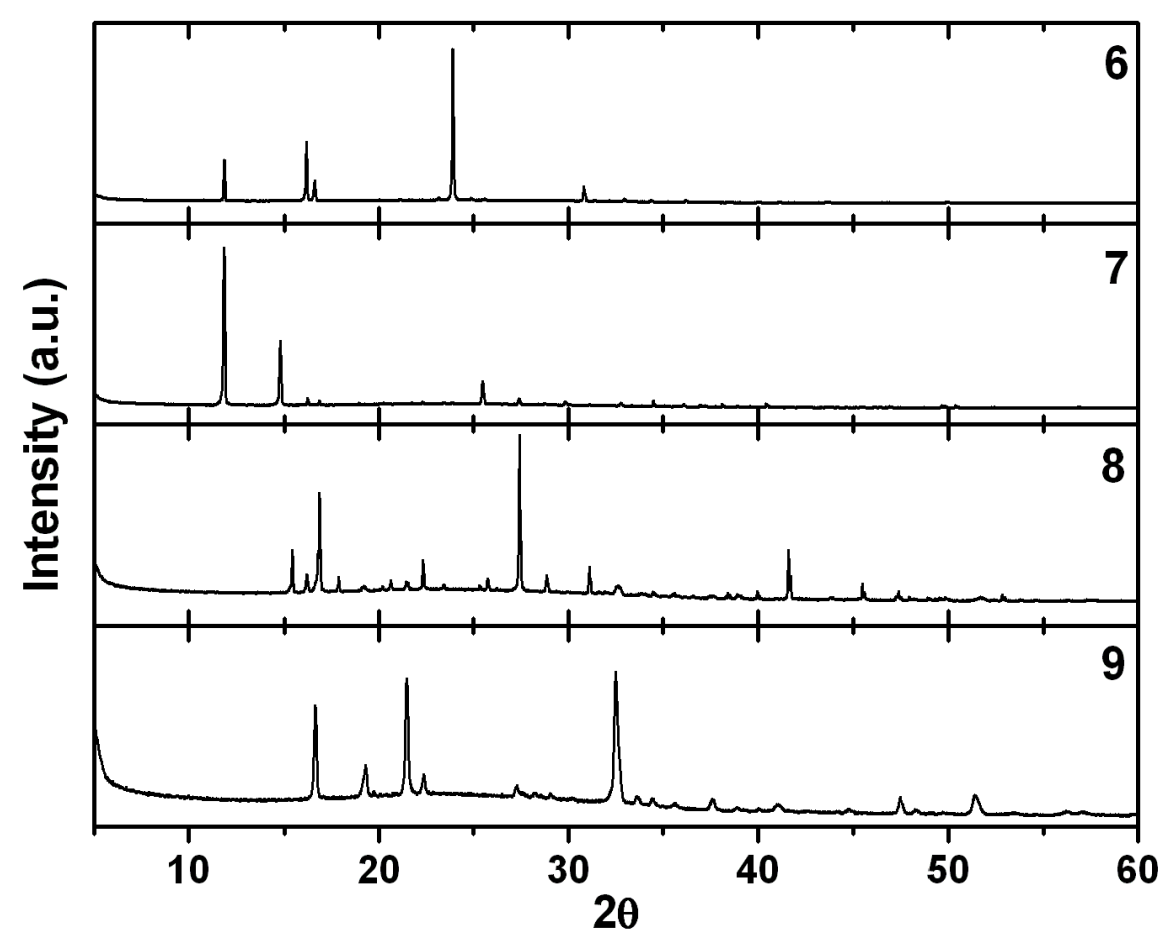


Figure S4. Variable Temperature X-ray diffraction (VT-XRD) for 3.

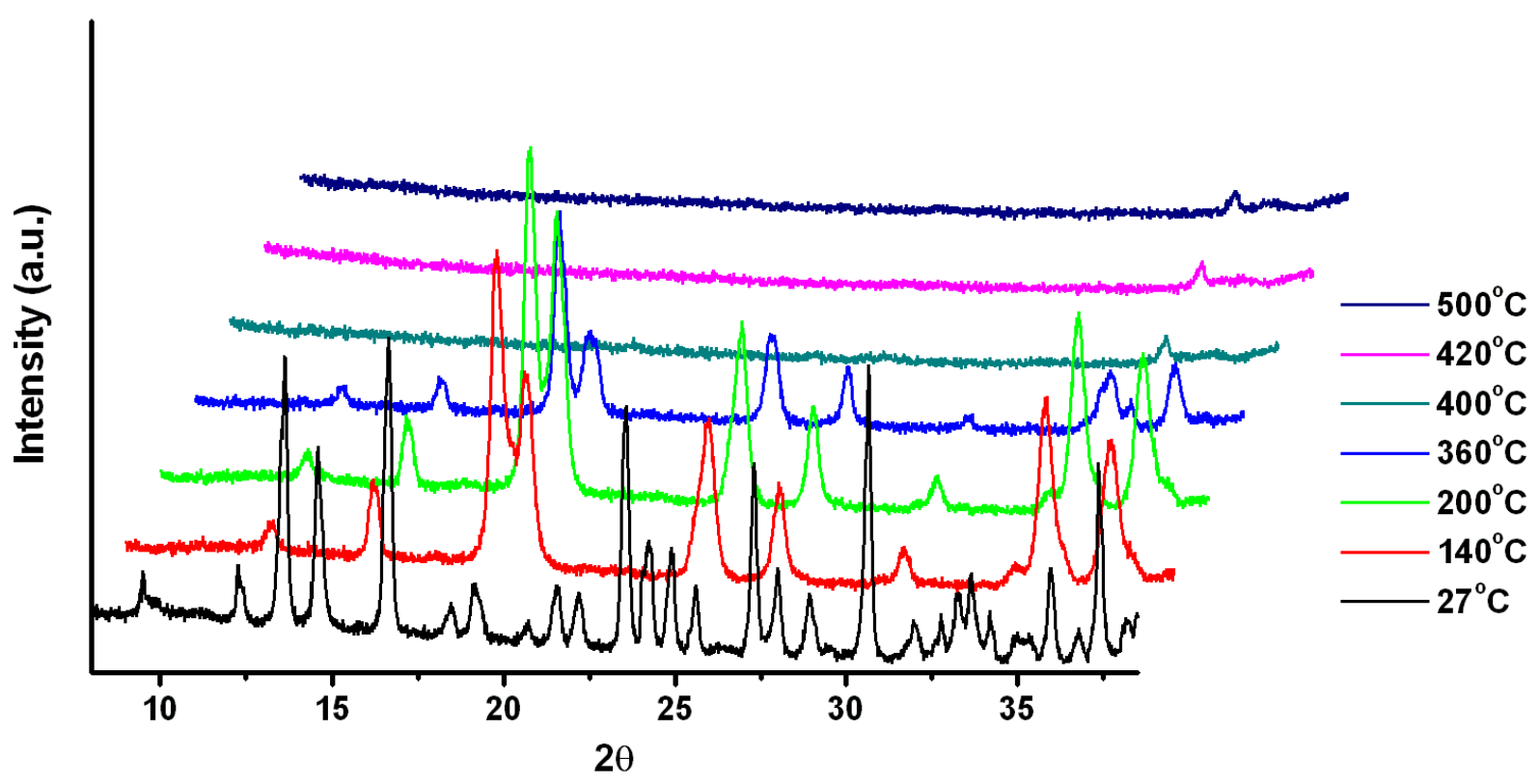




\section{Circular Dichroism}

Figure S5. Circular dichroism of aqueous solutions containing tartaric acid.

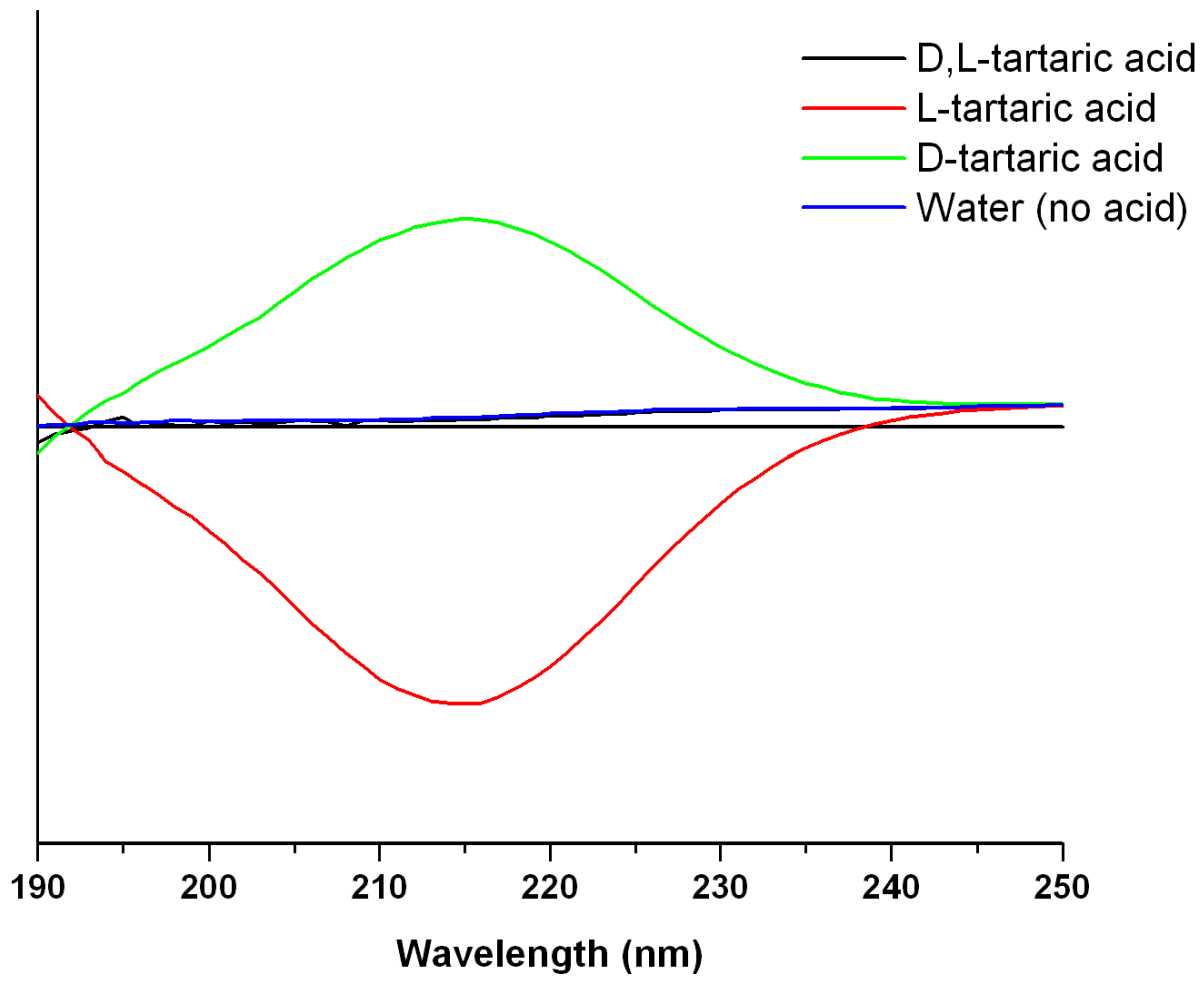


Figure S6. Circular dichroism of magnesium tartrates synthesized using D,L-tartaric acid at $180^{\circ} \mathrm{C}$ and $200^{\circ} \mathrm{C}$ for 2 days.

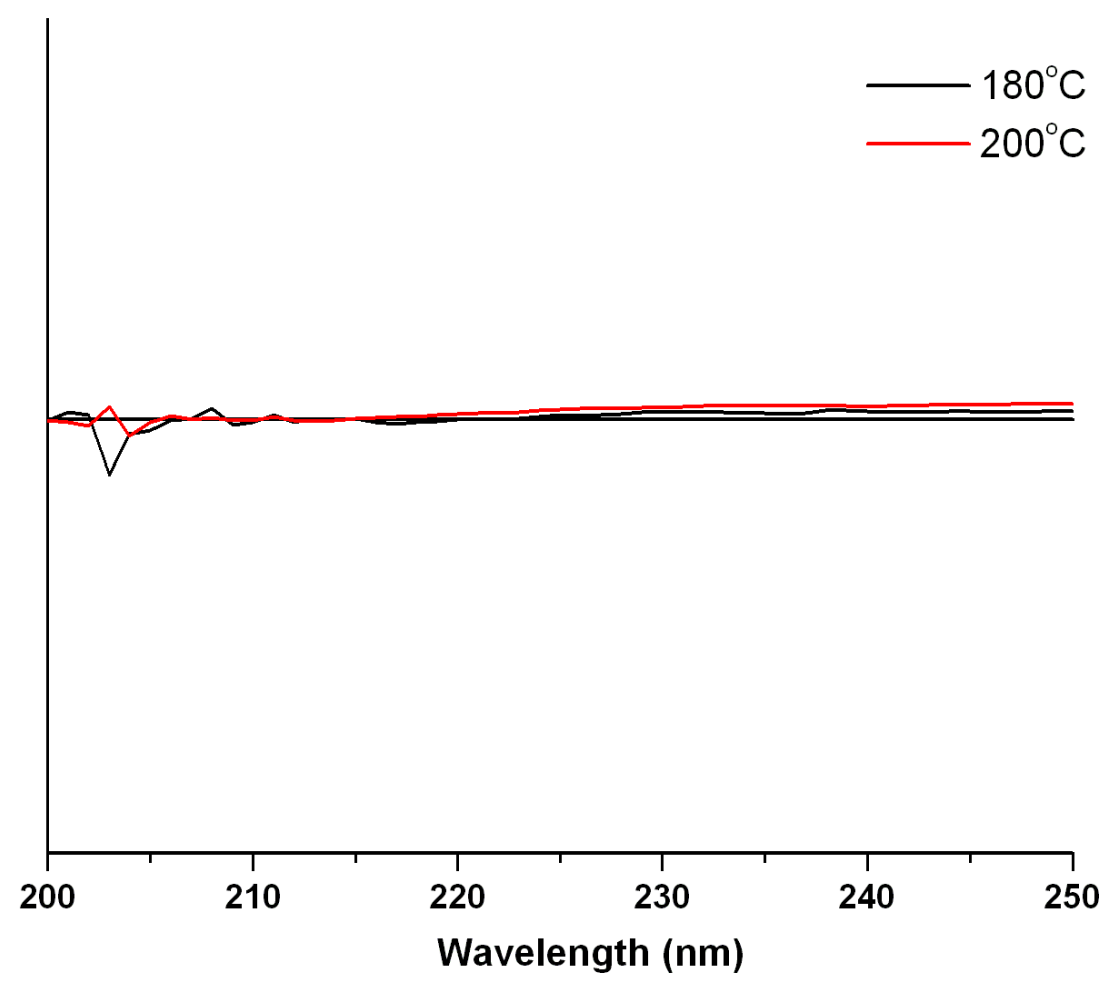


Figure S7. Circular dichroism of magnesium tartrates synthesized using D-tartaric acid at $150^{\circ} \mathrm{C}, 180^{\circ} \mathrm{C}$, and $200^{\circ} \mathrm{C}$ for 2 days.

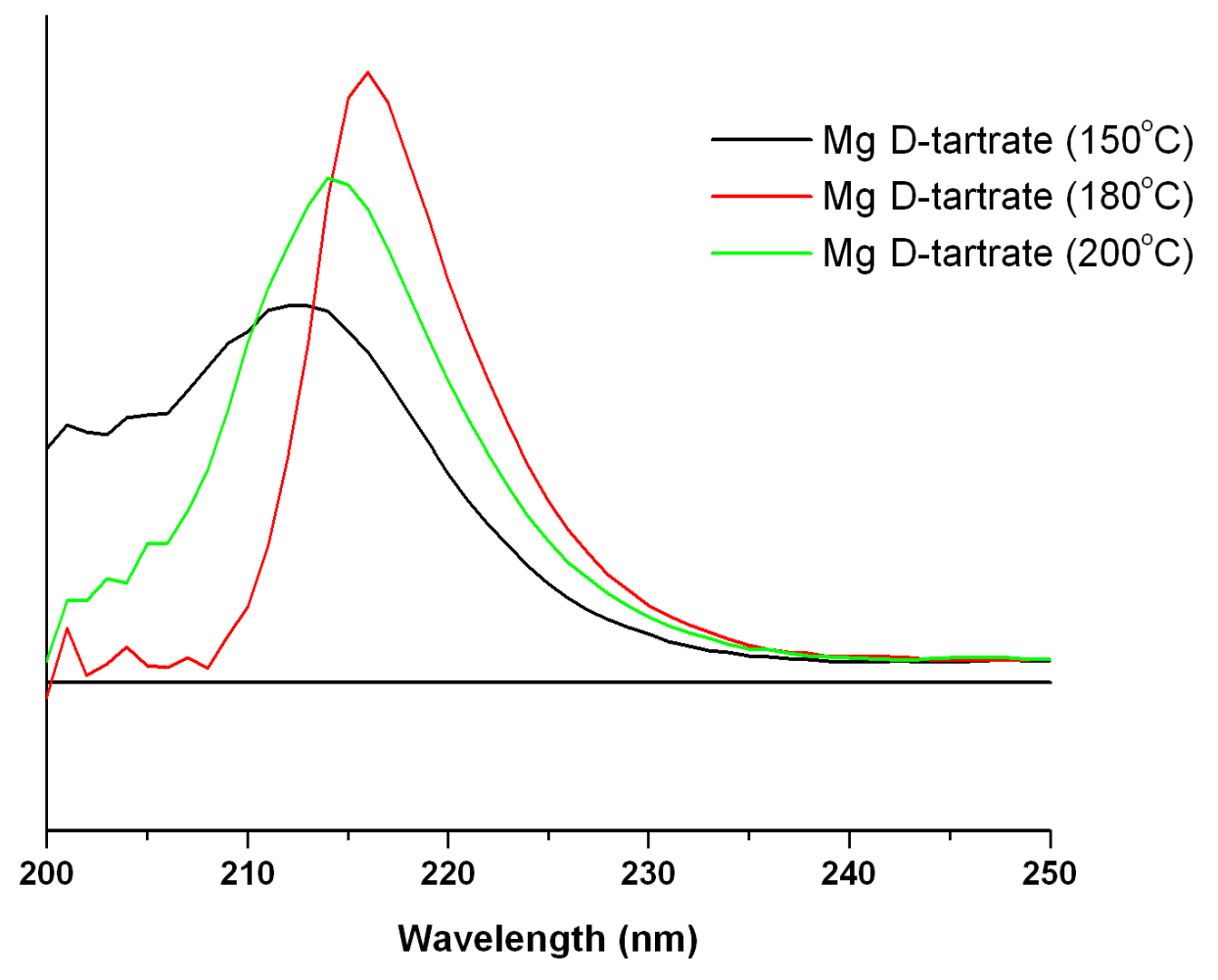

